\title{
Sensitivity to observations applied to FASTEX cases
}

\author{
A. Doerenbecher and T. Bergot \\ Météo-France, Centre National de Recherches Météorologiques, Toulouse, France \\ Received: 15 September 2000 - Revised: 28 February 2001 - Accepted: 17 April 2001
}

\begin{abstract}
The concept of targeted observations was implemented during field experiments such as FASTEX, NORPEX or WSRP in order to cope with some predictability problems. The techniques of targeting used at that moment (adjointbased or ensemble transform methods) lead to quite disappointing results: the efficiency of the additional observations deployed over sensitive areas did not turn out to remain consistent from one case to another. The influence of targeted observations on the forecasts could sometimes consist of strong improvements, or sometimes strong degradations. It turns out that the latter failure explains why the concept of optimal sampling arose. The efficiency of adaptive sampling appears to depend on the assimilation scheme that deals with the observations. It is then very useful to integrate the nature of the assimilation algorithm, as well as the deployment of the conventional network of observations (redundancy issues between targeted and conventional network) in the definition of the sensitive pattern to be sampled. Therefore, we chose the tool of the sensitivity to observations to allow us to test such an approach. The sensitivity to targeted observations (that utilizes the adjoint of the linearized NWP model and the adjoint of the assimilation operator) seems to be a suitable tool to obtain an insight into the tricky issue of the optimization of the sampling strategies.
\end{abstract}

To understand better the intrinsic patterns and the influence of the 3D-Var assimilation scheme on the sensitive structures to be sampled, we present here some detailed results on a FASTEX targeting case. We focus on the dropsondes deployed by the Gulfstream IV (jet-aircraft) along its first flight during Intense Observing Period 17 that started on the 17 February 1997 . The sensitivity to observation is used as a diagnostic tool for studing targeting from a critical point of view. It is shown that assimilation processes can have an important effect on the classical sensitivity fields, and particularly on their vertical extension. For example, in the studied case, the classical sensitivity fields remain at a lower level than $400 \mathrm{hPa}$, whereas the sensitivity to observations

Correspondence to: T. Bergot (Thierry.Bergot@meteo.fr) stretches up to $250 \mathrm{hPa}$. However, the maximum values can be found at approximately $700 \mathrm{hPa}$ in both sensitivity fields.

The studied case shows that the efficiency of observations depends not only on the sensitivity but also on the deviations between the observations and the background field. An example of the use of this diagnosis for comparing the relative efficiency of different kinds of observations is also presented. This work points out that it is very complicated to optimize the efficiency of adaptive observations, and that the assimilation of an entire set of observations (both conventional and adaptive network) needs to be considered.

\section{Introduction}

Despite numerous advances in the domain of numerical weather forecasting (improvement in data assimilation schemes and improvement in numerical models) during the past few years, the forecast of some meteorological events (like rapid cyclogenesis) remains a difficult problem. Since these meteorological situations have often tragic socioeconomic consequences, it is crucial to produce an accurate forecast of such events. These forecast errors partly result from inaccuracies in the initial conditions. These inaccuracies are a consequence either of the errors in the observations and in the background field, or of the inhomogeneous observation network, or of approximations made in the assimilation scheme used for interpolating observations and for producing initial conditions.

In order to try to improve the forecasting of such events, a new observational strategy has been proposed. The purpose was to add so-called adaptive or targeted observations to the conventional observing network in order to control the growth of forecast errors (Emanuel et al., 1995; Snyder, 1996). This observational network is adaptive in the sense that the location of these measurements varies from day-today (in opposition to the quasi-permanent conventional network of observations). This strategy was tested during recent field experiments: FASTEX (Joly et al., 1999), NORPEX 
(Langland et al., 1999), and WSRP (Szunyogh et al., 2000). The results from these field experiments show that the inclusion of targeted data can significantly improve the forecast in some cases. However, a large case to case variability exists, and it seems that the efficiency of targeted observations depends on numerous parameters (Bergot, 1999). One of the most important parameters is the assimilation scheme used for producing initial conditions. In fact, targeted observations have to go through the assimilation scheme, together with conventional data, to produce initial conditions, and their efficiency is strongly related to the accuracy of such data assimilation processes (Bergot, 2001; Bishop et al., 2001; Morss, 1999).

Sampling strategies, as tested during FASTEX, NORPEX or WSRP, do not appear to be optimal in the sense that there is no explicit care about redundancy (between targeted and conventional observations) and that the number, the nature, and the deployment of those adaptive observations do not guaranty a maximum improvement on the subsequent forecasts at the lowest cost. Further progress in targeting should consider such issues. Moreover, the effectiveness of the sampling of the sensitive area appears to depend on the assimilation scheme (Bergot, 2001). Therefore, it seems essential to include the effects of the assimilation processes in the way adaptive observations are defined (number, observed parameter, deployment). In this way, Baker and Daley (2000) have explored, in an idealized context, a new approach called sensitivity with respect to observations, hereafter called sensitivities to observations. These sensitivities to observations are defined in observation space, and point out the observations in which a given forecast aspect is sensitive. This tool makes it possible to highlight the effect of the assimilation scheme on adjoint-based sensitivity patterns. One of the advantages of sensitivity to observations is that it takes into account the existing conventional observations, as well as the way the targeted and conventional observations are assimilated.

The goal of this article is to illustrate the added value of sensitivity to observations with respect to classical sensitivity fields for real FASTEX targeted flights, and to explore the potential use of sensitivity to observations in the context of adaptive observations.

\section{Sensitivity to observations: principle}

The general theoretical principles of the sensitivity to observations are recalled here, and a more detailed formalism can be found in Baker and Daley (2000) or Doerenbecher and Bergot (2001, currently in progress). The sensitivity to observations will be applied within a variational assimilation context, and the notations are following Ide et al. (1997).

Let $\boldsymbol{x}_{a}(t)$ be the state vector, defined at time $t$, and called analysis. It is the output from the assimilation process which summarizes the information from all observations, $\boldsymbol{y}(t)$, and from the background field, $\boldsymbol{x}_{b}(t)$. Let $t_{0}$ be the initialization time, and $t_{1}$ be the final (or "verification") time at which a forecast aspect $S\left[\boldsymbol{x}_{f}\left(t_{1}\right)\right]=S\left[\mathcal{M} \boldsymbol{x}_{a}\left(t_{0}\right)\right]$ is computed, and let $\mathbf{M}$ be the linear approximation (or "tangent linear model") of the weather forecast model $\mathcal{M}$. Following the first order expansion of $S$, and following the definition of the adjoint model, $\mathbf{M}^{T}$, the sensitivity with respect to initial conditions, $\nabla_{\mathbf{x}} S$, is given by (see Rabier et al. (1996) or Errico (1997) for more details)

$\nabla_{\mathbf{x}} S=\mathbf{M}^{T} \partial S / \partial \boldsymbol{x}\left(t_{1}\right)$.

From the assimilation point of view, the initial conditions, $\boldsymbol{x}_{a}\left(t_{0}\right)$, are related to the background field, $\boldsymbol{x}_{b}\left(t_{0}\right)$, and to the observations, $\boldsymbol{y}\left(t_{0}\right)$, by (see Ghil and Malanotte-Rizzoli (1991) or Lorenc et al. (1986) for more details)

$\boldsymbol{x}_{a}\left(t_{0}\right)=\boldsymbol{x}_{b}\left(t_{0}\right)+\mathbf{K}\left[\boldsymbol{y}\left(t_{0}\right)-\mathcal{H}\left(\boldsymbol{x}_{b}\left(t_{0}\right)\right)\right]$

where $\mathcal{H}$ is the so-called observation operator which interpolates from model variables to observation points. $\mathbf{K}$ stands for the assimilation gain operator which combines the background error covariance matrix $\mathbf{B}$, the observation error covariance matrix $\mathbf{R}$, and the linearized observation operator H:

$\mathbf{K}=\left(\mathbf{B}^{-1}+\mathbf{H}^{T} \mathbf{R}^{-1} \mathbf{H}\right)^{-1} \mathbf{H}^{T} \mathbf{R}^{-1}$.

From a practical point of view, it should be noted that inverse matrix $\left(\mathbf{B}^{-1}+\mathbf{H}^{T} \mathbf{R}^{-1} \mathbf{H}\right)^{-1}=\mathbf{A}$ represents the so-called analysis error covariance matrix that depends on time $t$. In the context of adaptive observations, one generally considers the observational network to be composed of conventional observations (noted with a subscript $c$ in the following equations), and of a targeted component (noted with a subscript $t$ in the following equations). If errors in targeted observations are uncorrelated with errors in the conventional observations, the observation error covariance matrix, $\mathbf{R}$, corresponding to the whole network (noted with subscript $c t$ in the following equations) can be written as

$\mathbf{R}_{c t}=\left[\begin{array}{cc}\mathbf{R}_{c} & 0 \\ 0 & \mathbf{R}_{t}\end{array}\right]$

Likewise, the observation operator should be detailed according to the conventional and targeted observation systems, and $\mathbf{H}_{c t}$ can be written $\left[\mathbf{H}_{c} \mathbf{H}_{t}\right]$; it corresponds to a specific ordering of observed data according to type. Following this idea, one may write the sensitivity to conventional observations, $\nabla_{\mathbf{y} c} S$, and the sensitivity to targeted observations, $\nabla_{\mathbf{y} t} S$, as

$$
\begin{aligned}
{\left[\begin{array}{c}
\nabla_{\mathbf{y} c} S \\
\nabla_{\mathbf{y} t} S
\end{array}\right] } & =\mathbf{K}_{c t}^{T} \nabla_{\mathbf{x}} S=\left[\begin{array}{c}
\left(\mathbf{A}_{c t} \mathbf{H}_{c}^{T} \mathbf{R}_{c}^{-1}\right)^{T} \\
\left(\mathbf{A}_{c t} \mathbf{H}_{t}^{T} \mathbf{R}_{t}^{-1}\right)^{T}
\end{array}\right] \nabla_{\mathbf{x}} S \\
& =\left[\begin{array}{l}
\mathbf{R}_{c}^{-1} \mathbf{H}_{c} \mathbf{A}_{c t} \nabla_{\mathbf{x}} S \\
\mathbf{R}_{t}^{-1} \mathbf{H}_{t} \mathbf{A}_{c t} \nabla_{\mathbf{x}} S
\end{array}\right]
\end{aligned}
$$

where $\mathbf{A}_{c t}$ is the analysis covariance error defined for both the conventional and the targeted networks; it is worth recalling here that $\mathbf{A}$, as well as $\mathbf{B}$, and $\mathbf{R}$, are self-adjoint $\left(\mathbf{A}^{T}=\mathbf{A}\right.$ and $\left.\mathbf{R}^{-T}=\mathbf{R}^{-1}\right)$, so the superscript ${ }^{T}$ does not necessarily appear, such as in the right-hand side terms of Eq. (5). In those equations, the step that corresponds to 
the calculation of the classical sensitivity to initial conditions clearly appears. The effect of the assimilation scheme and of the conventional observations simultaneous to the adaptive ones is mainly contained in $\mathbf{A}_{c t}$. It is the keystone of this sensitivity approach.

Most of the current assimilation schemes are based on the variational method in which the analysis state is defined as the minimum of a cost function. This means that the analysis error covariance matrix $\mathbf{A}$ is not computed during the minimization. Nevertheless, such information is required for the calculation of the sensitivity to observations. In fact, we do not need to know the actual matrix of the variance-covariance of the analysis errors. What is needed is a projection of this matrix along the sensitivity to initial conditions $\nabla_{\mathbf{x}} S$. The 3D-Var assimilation scheme does not give any information about A, however, several methods exist for obtaining an estimate of it (Fisher and Courtier, 1995). Following this work, Doerenbecher and Bergot (2001, currently in progress) showed how to obtain an accurate estimate of this matrix in the unstable direction of the sensitivity in an operational 3DVar context. This method is applied here in the French operational weather forecast ARPEGE model (Courtier et al., 1991).

\section{Application to FASTEX cases}

\subsection{FASTEX framework}

A systematic survey of FASTEX targeted flights with 3DVar and 4D-Var assimilation systems (Bergot, 1999, 2001) demonstrated that the improvement of the forecasts is strongly case to case dependent. Moreover, these two studies and other studies based on simulated observations (Bergot et al., 1999; Bishop et al., 2001; Morss, 1999) described some difficulties in sampling and analyzing sensitive areas. In this sense, the sampling strategies of sensitive areas, as tested during FASTEX, do not appear to be optimal. Here, "optimal" means that the deployment of adaptive observations has to ensure a maximum improvement of the forecast with a minimum number of suitable dropsondes. Such observations consist of temperature, and wind and humidity measurements. FASTEX provides the first opportunity for examining the impact of targeted observations in an operational context, and numerous targeted observations are available (see FASTEX home page: http://www.cnrm.meteo.fr/fastex/). The data collected during this campaign can be useful for better understanding a posteriori why targeting implemented at that time did not produce the expected strong impact.

The sensitivity to observations is used here in a purely diagnostic mode (i.e. once the observations have been made). As shown hereafter, this approach can provide a powerful tool to obtain an insight on how targeted observations will influence forecast quality.

\subsection{Application to FASTEX IOP17}

\subsubsection{Meteorological context and data set}

The FASTEX IOP17 (Intensive Observing Period) case was an example of explosive cyclogenesis, with a strong deepening rate of $40 \mathrm{hPa}$ in 24 hours, and with the lowest central pressure (943 $\mathrm{hPa}$ at 00:00 UTC on the 20 February 1997) ever observed during FASTEX. A synoptic description of this IOP is given in Cammas et al. (1999).

A NOAA Gulfstream IV (hereafter GIV) flight mainly had a targeting goal in the earlier stage of the development of the cyclone (take off at 15:00 UTC and landing at 20:00 UTC on the 17 February 1997). Twenty dropsondes were deployed (see Fig. 1) inside the target area defined from the sensitivity fields which were operationally computed by both MétéoFrance and the Naval Research Laboratory. In the first part of the flight (northern and eastern legs of the flight), the sondes were released below $350 \mathrm{hPa}$, while along the southern flight track, the dropsondes were launched from above the tropopause, near $150 \mathrm{hPa}$.

\subsubsection{Sensitivity fields}

In a first step, the classical sensitivity to initial conditions is computed as during FASTEX: the forecast aspect $S$ is the enstrophy of the forecast $\boldsymbol{x}_{f}\left(t_{1}\right)$, vertically integrated between 950 and $790 \mathrm{hPa}$, and horizontally averaged over a verification area centered on the studied low $\left(60^{\circ} \mathrm{N}-45^{\circ} \mathrm{S}\right.$ and $\left.15^{\circ} \mathrm{W}-0^{\circ} \mathrm{W}\right)$. Using the French NWP ARPEGE model, the sensitivity is then computed with respect to the control variables, namely temperature, humidity, divergence, and vorticity. The assimilation scheme used is 3D-Var. The targeting time $t_{0}$ (17 February 1997 at $18 \mathrm{Z}$ ) corresponds to the analysis $\boldsymbol{x}_{a}\left(t_{0}\right)$, to the first guess $\boldsymbol{x}_{b}\left(t_{0}\right)$ and to the observations $\boldsymbol{y}_{c t}$. The forecasted trajectory is performed over 42 hours, so that the verifying time $t_{1}$ corresponds to the 19 February at 12:00 UTC. This range defines the optimization time for the classical gradient to initial conditions $\nabla_{\mathbf{x}} S$.

The computation of the sensitivity field is similar to what was done during FASTEX. However, it should be noted that the sensitivity calculations are based on the trajectory issued from the analyzed initial conditions at targeting time $t_{0}$. This is a major difference between the diagnostic context used here and the operational one, in which the trajectory was necessarily computed from some forecast initiated well before the targeting time. However, for the studied case, the use of either a diagnostic or an operational based trajectory does not lead to many differences inside the location of sensitive areas.

The classical sensitivity to initial conditions, $\nabla_{\mathbf{x}} S$, is computed as detailed above, and is compared to the sensitivity to targeted observations, $\nabla_{\mathbf{y} t} S$. This comparison allows us to better understand the influence of the assimilation processes on the sensitivity fields. Figures 2a, 3a, and 4a show the sensitivity to initial conditions projected onto the targeted observation space, $\mathbf{H}_{t} \nabla_{\mathbf{x}} S$, for the $T, U$ and $V$ observed param- 


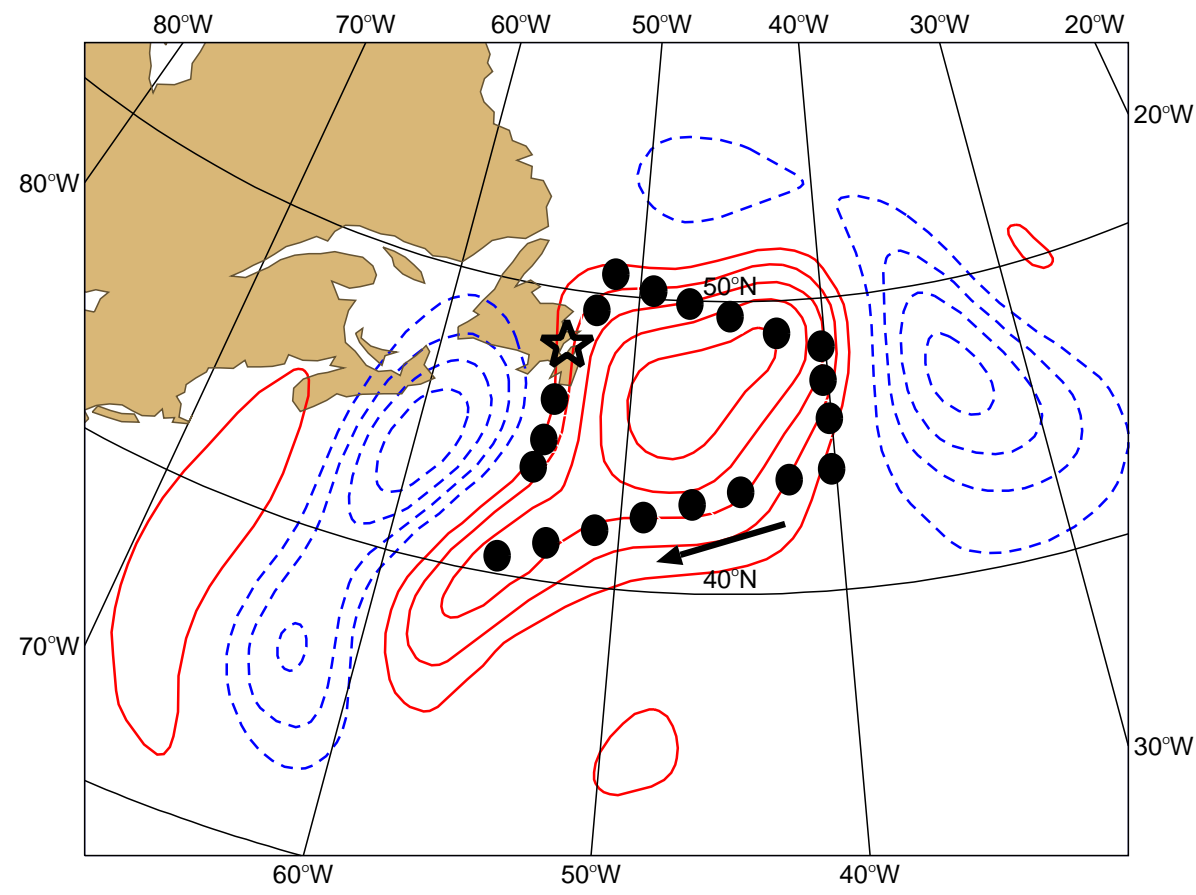

Fig. 1. Gulfstream IV flight for the IOP17, 15:00 UTC-20:00 UTC on 17 February 1997 . Black dots indicate the dropsonde positions. The solid arrow indicates the direction of the flight and the star indicates the location of the takeoff and landing at St-Johns, Newfoundland. The classical sensitivity field to initial conditions of temperature at $700 \mathrm{hPa}$, as defined during FASTEX, is plotted as a background. eters. These sensitivity fields are plotted as cross-sections along the flight: the vertical axis represents the pressure level of the measurement, and the dropsonde locations are plotted in the horizontal axis, according to their distance. It is worth recalling that $\mathbf{H}_{t} \nabla_{\mathbf{x}} S$ and $\nabla_{\mathbf{y} t} S$ are defined in the observation space and that sensitivity values are computed at observation points. This implies some difficulties for a suitable graphical representation. In the following figures, values on observation space were interpolated in order to be able to draw a set of isolines. This graphical choice allows one to clearly depict the patterns of sensitivity. One can notice strong sensitivity to initial conditions in the northern and eastern part of the flight, for both temperature and wind. The maximum of sensitivity is located at a low level, around $700 \mathrm{hPa}$, for the dropsonde \#5 in the case of temperature, and for the dropsonde \#6 in the case of the $U$-wind and $V$-wind.

Figures $2 b, 3 b$, and $4 \mathrm{~b}$ show the sensitivity with respect to observations, $\nabla_{\mathbf{y} t} S$, for temperature, $U$-wind and $V$-wind. As previously explained, these sensitivity fields are plotted as cross-sections along the flight. Both $\nabla_{\mathbf{x}} S$ and $\nabla_{\mathbf{y} t} S$ exhibit high values in the northern part of the flight. The maximum sensitivity to observations for temperature is always located at the dropsonde \#5 near $700 \mathrm{hPa}$. The assimilation processes have a weak effect on the location of these strong sensitivities. A comparison of Fig. 2a and Fig. 2b shows that the major difference between the patterns of sensitivity to initial conditions and sensitivity to observations is their vertical extension. While $\nabla_{\mathbf{x}} S$ remains mostly confined in the lowest levels of the atmosphere (below $500 \mathrm{hPa}$ ), $\nabla_{\mathbf{y} t} S$ extends throughout the whole atmosphere. This is also clear for $U$-wind (Fig. 3a and Fig. 3b). This increase in the vertical extension of the sensitivity field is a consequence of the ver- tical structure functions (vertical correlations) used in the assimilation (see Appendix A). The same remark can be made when the classical sensitivity to initial condition (Fig. 4a) is compared to the sensitivity with respect to the $V$-wind observations (Fig. 4b). In the first case, the maximum sensitivity remains at a level lower than $650 \mathrm{hPa}$. Although strongest sensitivities are found at lower levels, when both $\nabla_{\mathbf{y} t} S$ and $\nabla_{\mathbf{x}} S$ are examined, significant values of $\nabla_{\mathbf{y} t} S$ can appear up to $350 \mathrm{hPa}$, due to the vertical spreading of $\nabla_{\mathbf{x}} S$.

As shown in Thépaut et al. (1996), structure functions can easily be illustrated by single observation assimilation experiments. Therefore, such an experiment has been performed to illustrate the former point using a single wind observation at $600 \mathrm{hPa}$. In this specific case, the increment represents the structure function associated with the simulated observed parameter. The structure function can be associated with a column of the matrix $\mathbf{A}$. It describes the covariances between a given model parameter (or the closest to the observed parameter) with all other parameters. Such a single observation experiment allows one to obtain an insight into a slice of $\mathbf{A}$.

The corresponding final increment, plotted in Fig. 5, exhibits a classical barotropic structure, keeping in mind that the 3D-Var assimilation scheme is used here. Moreover, one can notice the strong vertical correlation between the wind at the observed level and the surrounding wind field: for one observation located at $600 \mathrm{hPa}$, the depth over which the increment is higher than half of the increment at $600 \mathrm{hPa}$ is at about $650 \mathrm{hPa}$. For the FASTEX case detailed in this section, the inclusion of the assimilation processes in the sensitivity approach leads to some significant changes in the sensitivity patterns. If the classical sensitivity is confined below $500 \mathrm{hPa}$ (the highest significant sensitivity maximum is lo- 
cated at $600 \mathrm{hPa}$ in the northern part of the flight), the sensitivity to targeted observations exhibits a strong extremum at $600 \mathrm{hPa}$, but also local extrema at levels as high as $300 \mathrm{hPa}$. The latter clearly appears for the dropsonde \#15 for all parameters, especially temperature. This vertical stretching of sensitivity is interpreted as the effect of the structure functions described in operator $\mathbf{A}_{c t}$ (see the Appendix A for more details).

These results suggest that the assimilation scheme can have a strong influence on the structure of the sensitivity fields, and particularly on the vertical extension of the sensitive area.

\subsubsection{Impact of targeted observations}

The actual impact of targeted observations depends not only on the sensitivity fields, $\nabla_{\mathbf{y} t} S$, but also on the amplitude of the innovation vector, $\boldsymbol{y}_{t}-\mathcal{H}_{t}\left[\boldsymbol{x}_{b}\left(t_{0}\right)\right]$. For example, if the observed value is similar to the background value, then the impact will be small, even if the observation is located inside an area of strong sensitivity. To study this point, we focus here on the so-called impact function which is directly derived from the definition of sensitivity, as in Rabier et al. (1996). In our particular case, the perturbation is defined as the innovation vector, $\boldsymbol{y}_{t}-\mathcal{H}_{t}\left[\boldsymbol{x}_{b}\left(t_{0}\right)\right]$, and the linear estimate of the variation of the forecast aspect $S$ is given by

$\delta S_{o}=\left(\nabla_{y o} S\right)^{T}\left[\boldsymbol{y}_{o}-\mathcal{H}_{o}\left(\boldsymbol{x}_{b}\left(t_{0}\right)\right)\right]$

where subscript " $o$ " denotes a given type of observation. With such a computation based on the linear hypothesis, we directly obtain an estimate of the influence of a given kind of observation on the forecast aspect $S$. This impact depicts the actual influence of each observation, or group of observations, on the analysis and the subsequent forecast, as soon as they become part of the whole observational network.

Using the same graphical conventions as in Fig. 2, Fig. 6 shows the contribution from each temperature observation (Fig. 6a), and from each wind (combined $U$ and $V$ components) observation (Fig. 6b). The respective contributions from each wind component to the impact function are given in Figs. 7a and 7b, respectively. This impact combines the signs of the innovation and of the sensitivities to targeted observations. It points out small structures that contribute sometime in an opposite manner, even for nearby measurements, to the variation $\delta S$. In spite of some organized tilted structures that could be detected in the innovation (not shown), their combination with the sensitivity to the observations produces small-scale patterns, especially in the southern part of the flight. In the northern part, we detect a tilted structure. This is particularly noticeable for the temperature in Fig. 6a with a quite uniform field below it and strong contributions above it, but within a thin layer. Such opposite local contributions which can be found on the vertical of a given dropsounding may cancel out when the total contribution of that dropsounding to the impact function is considered. (a)

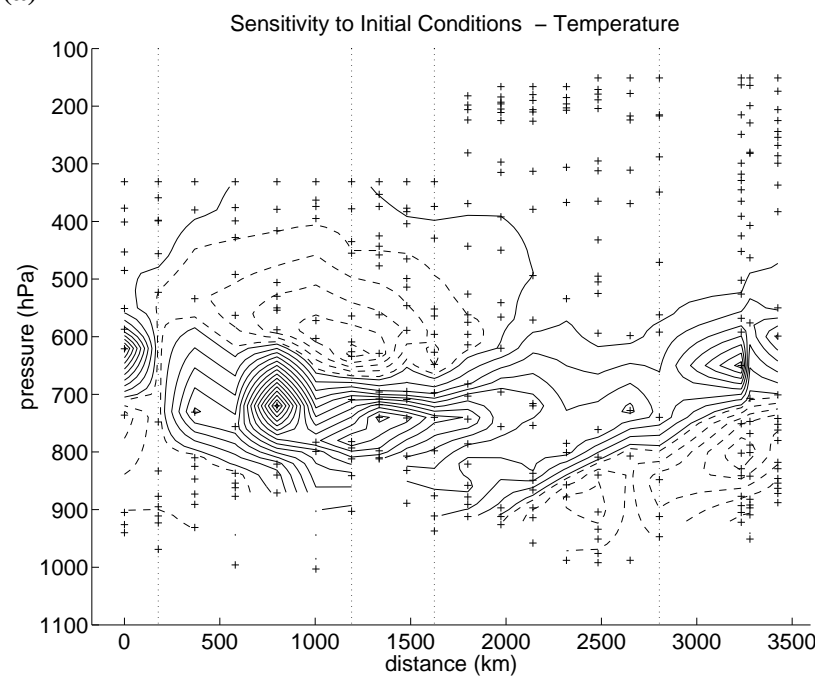

(b)

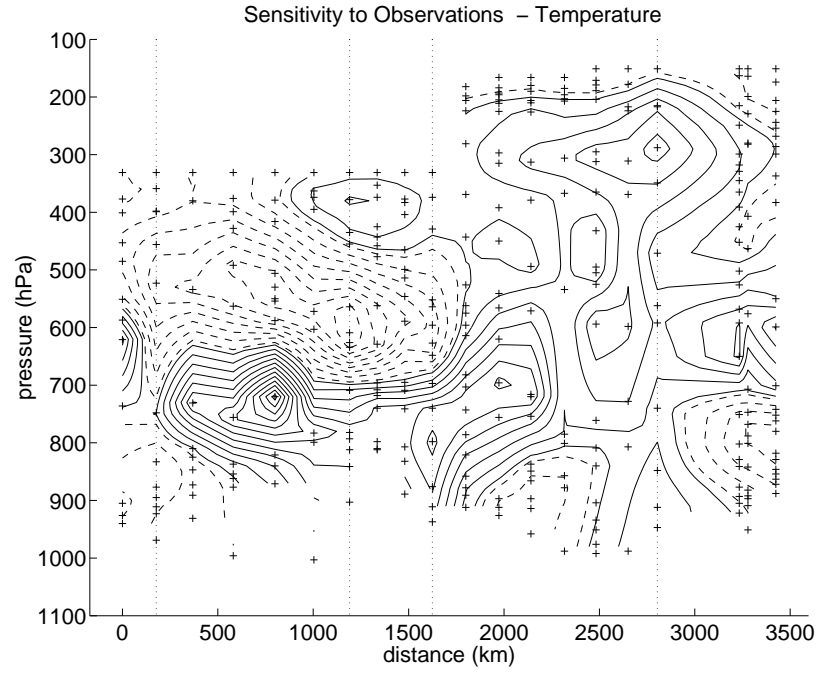

Fig. 2. GIV flight for IOP17. Sensitivity with respect to initial conditions, projected onto the temperature observation space (a) and sensitivity with respect to the observations of temperature (b). The vertical axis represents the pressure level of the measurement and the dropsonde locations are plotted in the horizontal axis according to their distance from flight departure point (St-Johns, Newfoundland). The crosses represent the location of the temperature measurements, and the vertical dotted lines represent the changes in direction during the flight.

Due to of the practical implementation of targeting which is achieved by means of dropsondes, it seems quite natural to gather this impact information from each dropsonde by integrating the contributions of each temperature and wind measurement. It then appears that some contributions will compensate each other. Therefore, a given dropsonde will not necessarily have an overall strong contribution if one of its single measurements has a strong impact.

It is then possible to separate the contributions from targeted dropsonde data and from conventional RAOBS data or 
(a)

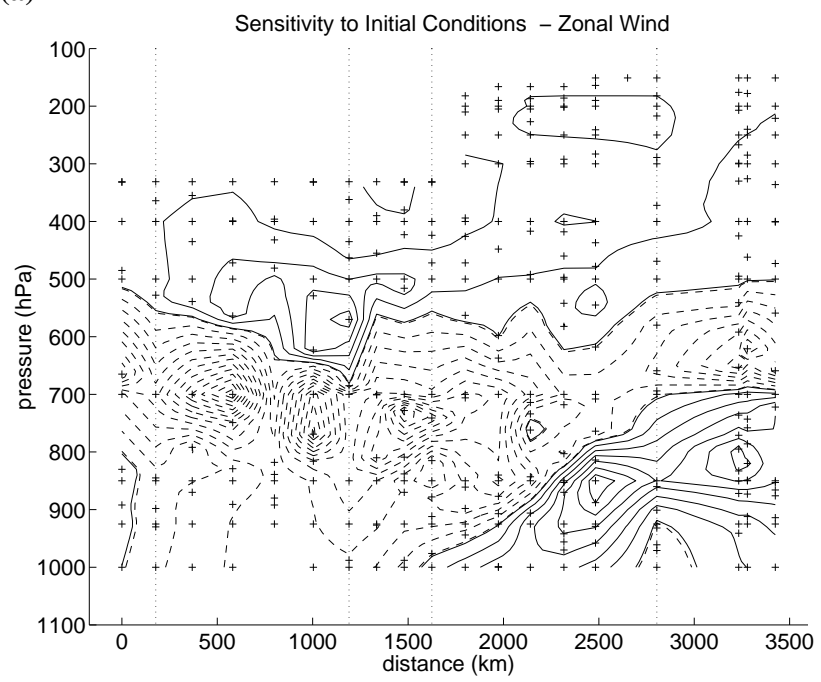

(b)

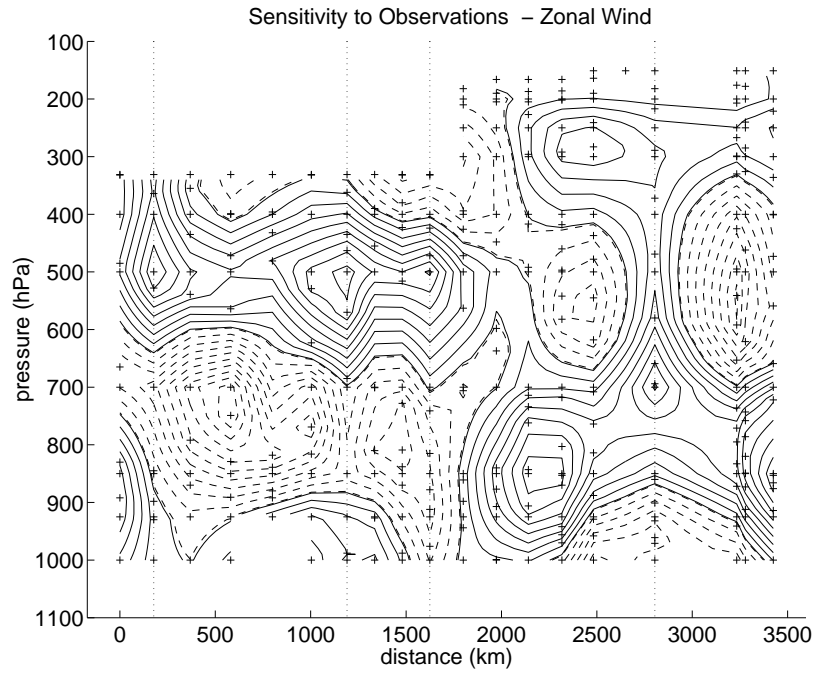

Fig. 3. Same as in Fig. 2, but for the observations of $U$-wind.

satellite data (SATOB in this study), and to compare their relative efficiency and influence on the forecast aspect $S$. Unfortunately, the innovation vector, $\boldsymbol{y}(t)-\mathcal{H}\left[\boldsymbol{x}_{b}\left(t_{0}\right)\right]$, and therefore the impact function, $\delta S$, can only be known once the observations are made. However, the comparison between the sensitivity and the impact can tell us whether the influence from observations is a consequence of either strong errors in the initial conditions, i.e. large differences between $\boldsymbol{y}(t)$ and the background $\boldsymbol{x}_{b}\left(t_{0}\right)$ combined with weak sensitivities, or strong sensitivities despite a weak innovation.

Figure $8 \mathrm{a}$ and Fig. $8 \mathrm{~b}$ show the extremum of the impact function for each dropsounding, in terms of temperature and wind, respectively. Strong impacts are always present in the northern part of the flight, for both temperature and wind. These strong impacts correspond to a strong sensitivity to initial conditions and to observations, as previously shown (see Figs. 2 to 4). For the studied case, one can also notice that the impacts for wind are generally stronger than (a)

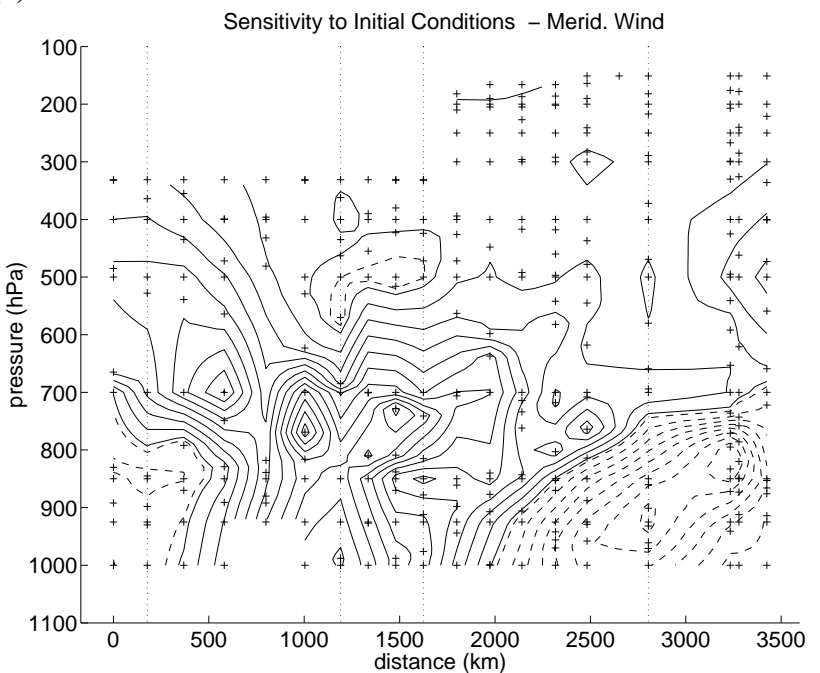

(b)

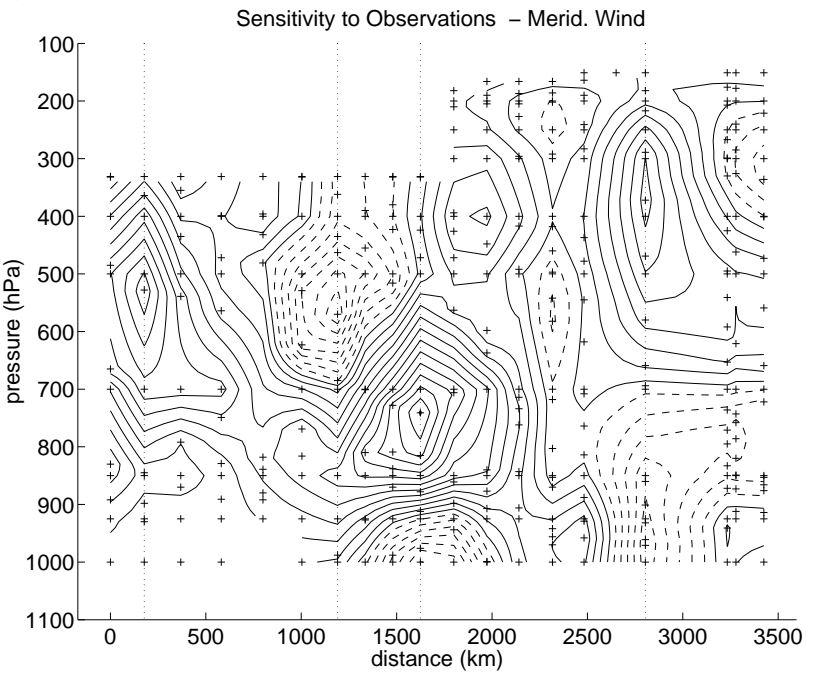

Fig. 4. Same as in Fig. 2, but for the observations of $V$-wind.

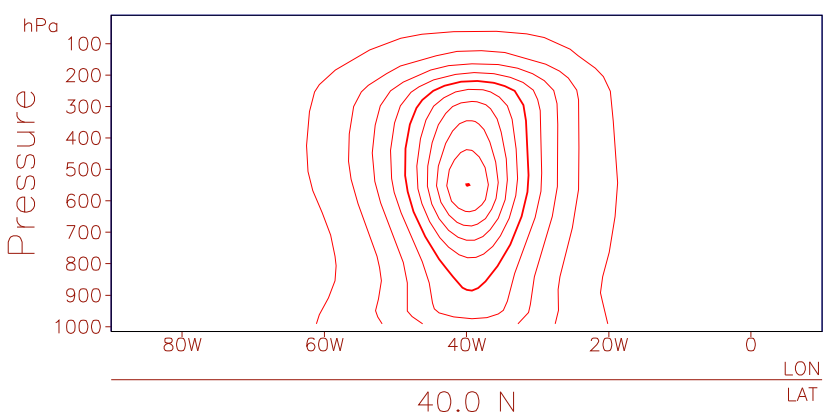

Fig. 5. Vertical correlation for assimilation of a single wind observation at $600 \mathrm{hPa}$. The bold line corresponds to half of the maximum value of the wind increments.

the impacts for temperature, for a given dropsounding. The extremum generally have opposite signs: a decrease of the forecast aspect $S$ for wind is associated with an increase 
(a)

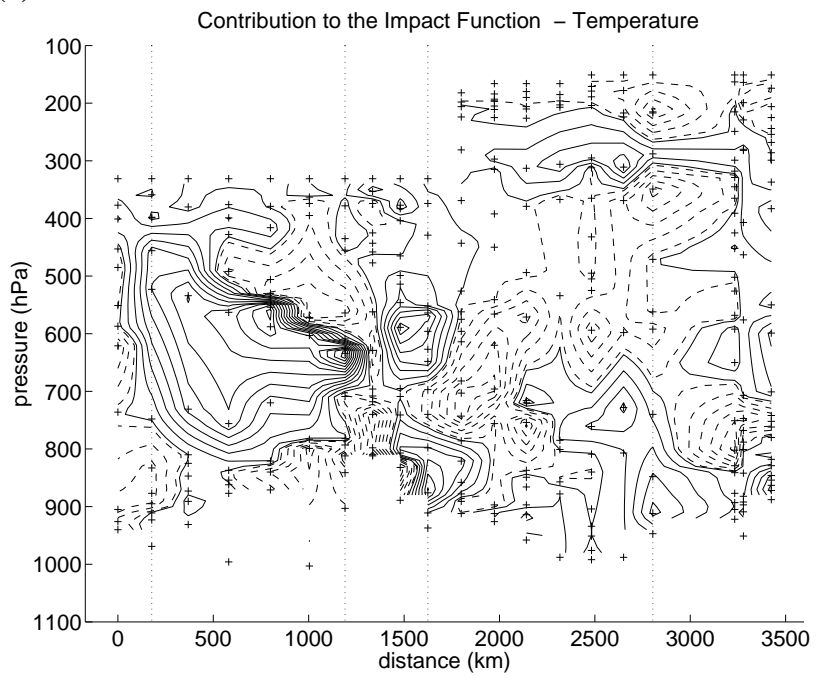

(b)

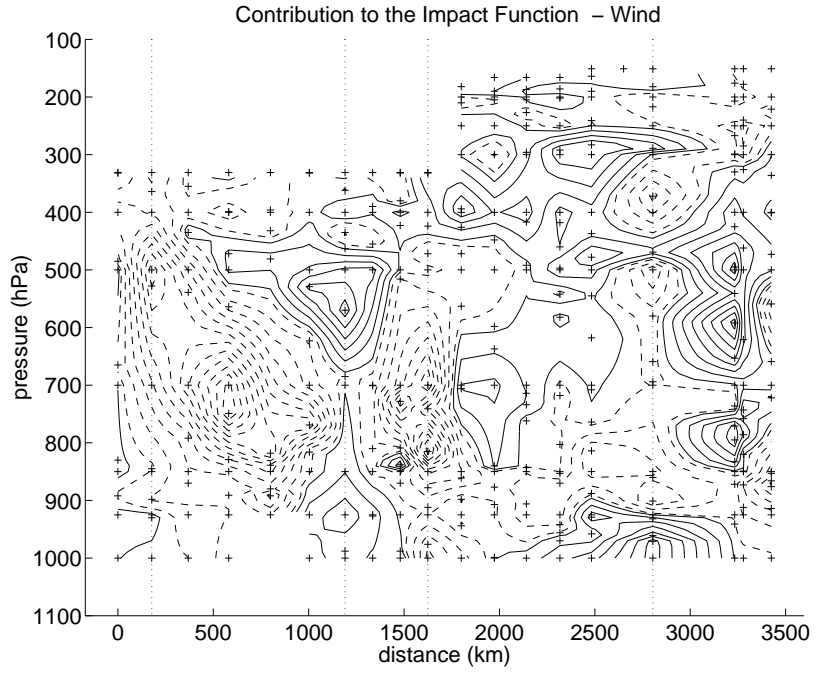

Fig. 6. Same graphical principle as in Fig. 2, but for the contributions $\delta S_{i}$ of each targeted temperature observation (a) and of each targeted couple of wind observations (b).

in $S$ for temperature. Figure 8b also shows that the impact function exhibits strong extrema in the southeastern part of the flight. Dropsonde \#10 does not correspond to any maximum of sensitivity to initial conditions, but sensitivity values remain high, especially at low levels $(800-750 \mathrm{hPa})$ (see Figs. 2 to 4). The examination of the sensitivity to observations clearly suggests that the $V$-wind component plays a major role. For dropsonde \#10 (Fig. 4b), a maximum of sensitivity is found at $750 \mathrm{hPa}$. This maximum of $\nabla_{\mathbf{y} t} S$ is embedded in an area with significant negative values of innovation, $\boldsymbol{y}_{t}-\mathcal{H}_{t}\left[\boldsymbol{x}_{b}\left(t_{0}\right)\right]$ (not shown), which produces the strongest $V$-wind contribution to $\delta S$ (Fig. 7b).

When focusing on the whole flight and considering all observed parameters, it appears that most of the strong contributions originate from wind measurements, i.e. the case for dropsonde numbers 2, 4, 10 and 17 . The strong contribu- (a)

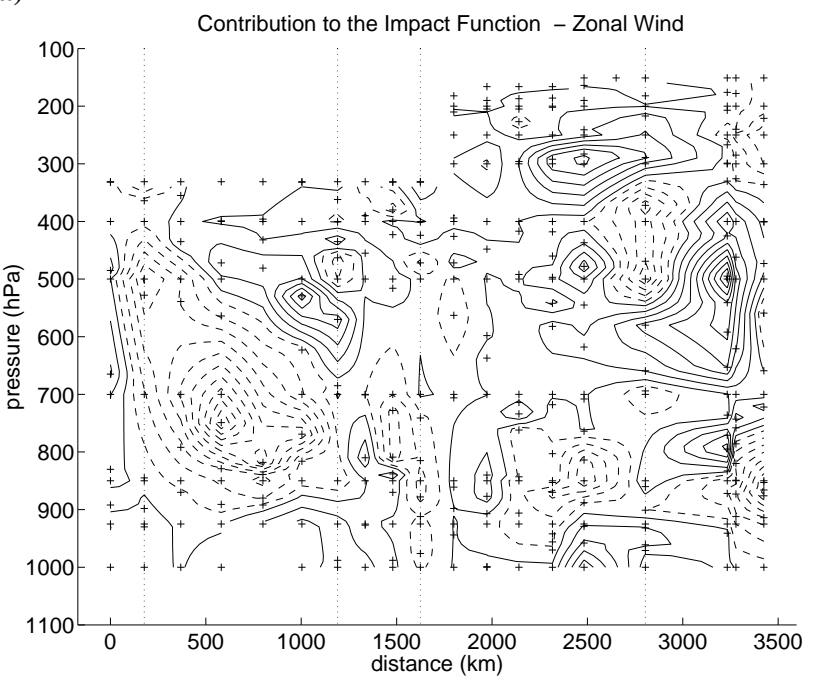

(b)

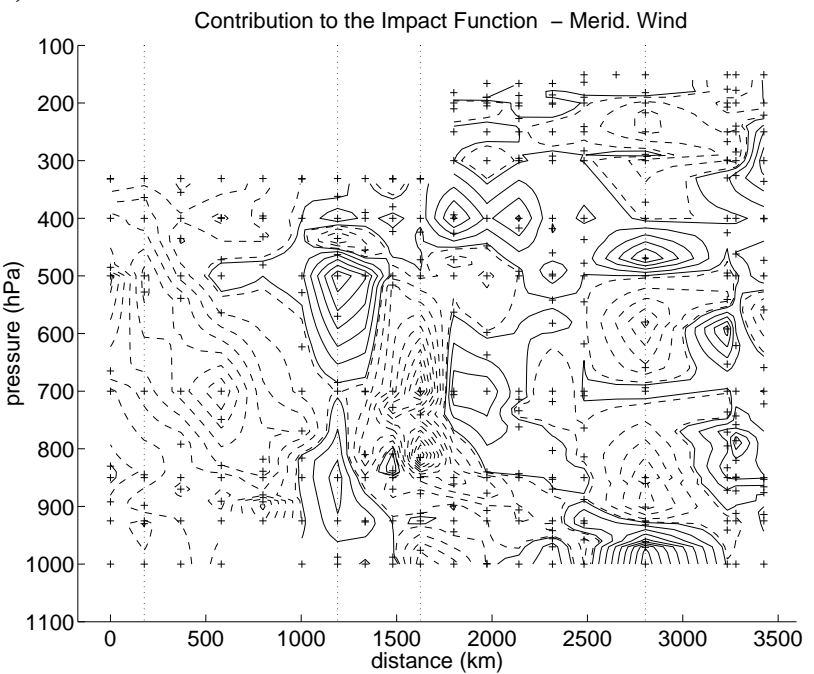

Fig. 7. Same graphical principle as in Fig. 2, but for the contributions $\delta S_{i}$ of each $U$ component of wind targeted observations (a) and of each targeted $V$ component of wind targeted observations (b).

tions of dropsonde numbers 2, 4 and 10 come from the conjugate effect of both $U$-wind and $V$-wind based contributions. In the case of the dropsonde \#17, it is essentially a $U$-wind based contribution; the sensitivity to $V$-wind is quite low in this area. As far as temperature is concerned, this parameter plays a role only in the northeastern part of the flight.

The strong impact of dropsonde \#4 for wind, and dropsonde \#8 for temperature are a consequence of strong discrepancies between the observations and the background field. With the sensitivity to observations being moderately high (relative to its maximum which is located at another observation point), the innovation is responsible for those fairly heavy contributions to $\delta S$.

Since we have a good confidence in the targeted observations, those discrepancies can be interpreted as errors in 
the background field. Moreover, it is helpful to recall here that the sensitivity never indicates where initial errors are located, and that the size of the errors should also be taken into account. However, it seems that for the studied cases, strong sensitivity is associated with a strong impact (but strong impact is not always associated with strong sensitivity).

The modification of the forecast aspect, $S$, due to the inclusion of targeted observations is the vertical summation of elementary impacts from each measurement at each level. To study the efficiency of targeted observations, these summations are plotted in Fig. 8c and Fig. 8d for temperature and wind (both $U$ and $V$-wind), respectively. For the studied case, the efficiency of targeted observations is clearly stronger for wind than for temperature. The wind and temperature impacts have often opposite effects. Figure 9 shows the efficiency of each dropsounding for all measurements and all levels. There is a strong correlation between Fig. 9 and Fig. 8d. This shows, for the selected case, the predominance of wind over temperature measurements in the efficiency of targeted observations. One can also notice that dropsoundings can have opposite effects: for example, the northern part of the flight leads to a decrease in $S$, while the southwestern part of the flight leads to an increase in it. Even nearby observations can have strong opposite effects. This example illustrates that it is very complicated to study and to optimize the efficiency of targeted observations. Moreover, it has been shown by Bergot (2001) that the influence of targeted flights on the forecasts is characterized by a large spread among cases: strong impacts as well as quite undetectable impacts were identified. In the latter case (weak impact), this characteristic of the flight does not mean that an observation by observation examination would depict any weak contribution for each observation. We can notice some strong opposite contributions to the variation of the impact. In such cases, the effect of the flight itself will be small, as all the contributions of measurements are summed up.

Another advantage of the sensitivity to observations associated with the calculation of the impact is that it can be used for comparing the relative efficiency of different kinds of observations. Figure 10 shows an example for the FASTEX IOP17 case. The targeted observations, plotted as TEMP messages, have the strongest impact, despite their low number ( 20 dropsondes). This impact is similar to the one from the entire set of conventional observations. This kind of diagnoses allows one to say that the targeted flight for FASTEX IOP17 is relatively efficient (weak number of dropsondes, yet strong impact). Similar results have been obtained by Bergot (2001) and Langland et al. (1999). Figure 10 also demonstrates that different kinds of observations can have opposite effects on the forecast aspect $S$. For example, the PILOT observations (measurement of wind) have an effect, in absolute value, similar to the SYNOP observations (surface measurements), but with an opposite sign. Therefore, the PILOT + SYNOP observations have no impact on the forecast aspect, despite relatively large individual contributions. It must be emphasized that the contribution to the impact of a given subset of observations (used for computing the initial conditions of the forecast) is valid only due to the presence in the analysis of all the other observations: the actual contribution of the subset to the impact will not be equal to the impact obtained if only this subset were used in the assimilation.

\section{Conclusions}

The recent concept of adaptive observations has been tested during the FASTEX field experiment carried out in JanuaryFebruary 1997. The goal of this concept is to add targeted observations inside sensitive areas in order to locally improve the initial conditions, and therefore the subsequent forecast. Different works have shown that the efficiency of targeted observations depends on numerous parameters, and particularly on the assimilation scheme used. In this way, it seems essential to include the assimilation processes in the definition of adaptive observations.

Following the preliminary work of Baker and Daley (2000), the concept of sensitivity to observations has been implemented in the French operational NWP model ARPEGE. The sensitivity to observations is defined in the observation space, and points out the observations to which a given forecast aspect is sensitive. In this study, this new tool is used in a diagnostic way (i.e. once the observations are made) for assessing the efficiency of FASTEX targeted observations for IOP17.

The results demonstrate that the assimilation scheme has a strong influence on the vertical structure of the sensitivity fields, with a significant increase in their vertical extension. This tool is also utilized for comparing the efficiency of different observations, with the calculation of the so-called impact function. This impact function represents the modification of the forecast aspect, $S$, due to given observations, under the linear hypothesis. For the studied case, strong sensitivity is generally associated with strong impact. Nevertheless, the contrary is wrong, and strong impact does not always correspond to strong sensitivity.

For this targeted flight, the efficiency of adaptive observations is clearly a consequence of the GPS wind measurements from dropsondes, and the temperature measurements have a weaker effect (about a fifth of the first for the considered $\delta S$ ). Even nearby observations can have opposite effects on the final variation of the forcast aspect $S$. If the gathered contributions of all the observations of a targeted flight result in a weak contribution to the variation of $\delta S$, it does not imply that each observation or group of observations have also a weak contribution on itself. This studied case clearly shows that it will be very complicated to optimize a priori (i.e. before the observations are done) the efficiency of targeted observations.

This work advocates the use of sensitivity to observations in order to study the efficiency of observations in an a posteriori context (i.e. once the observations are made). An important question now is to investigate the potential of this tool in a prognostic mode in order to try to optimize the efficiency of targeted observations. What will 
(a)

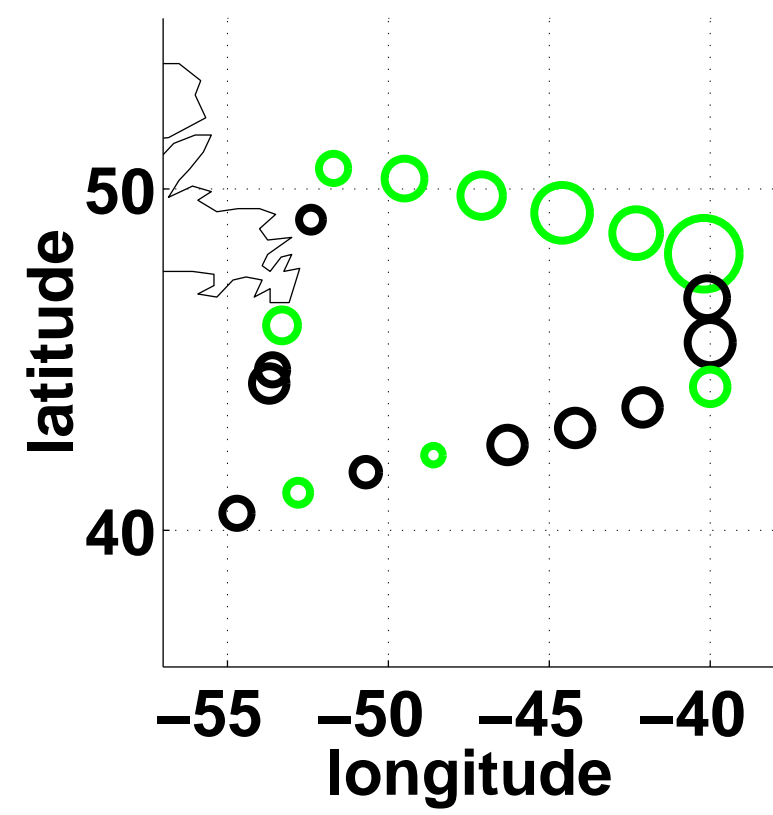

(b)

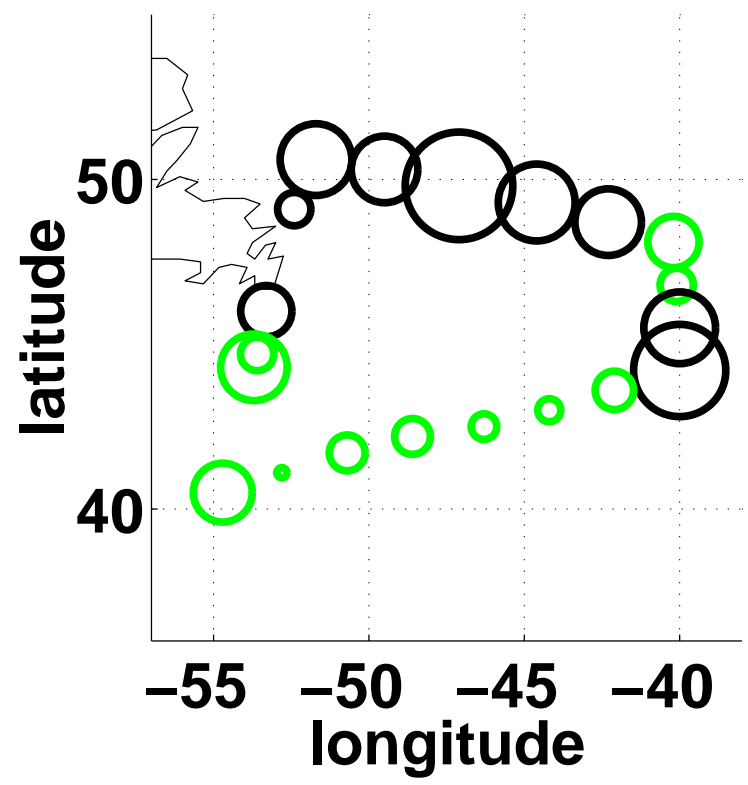

(c)

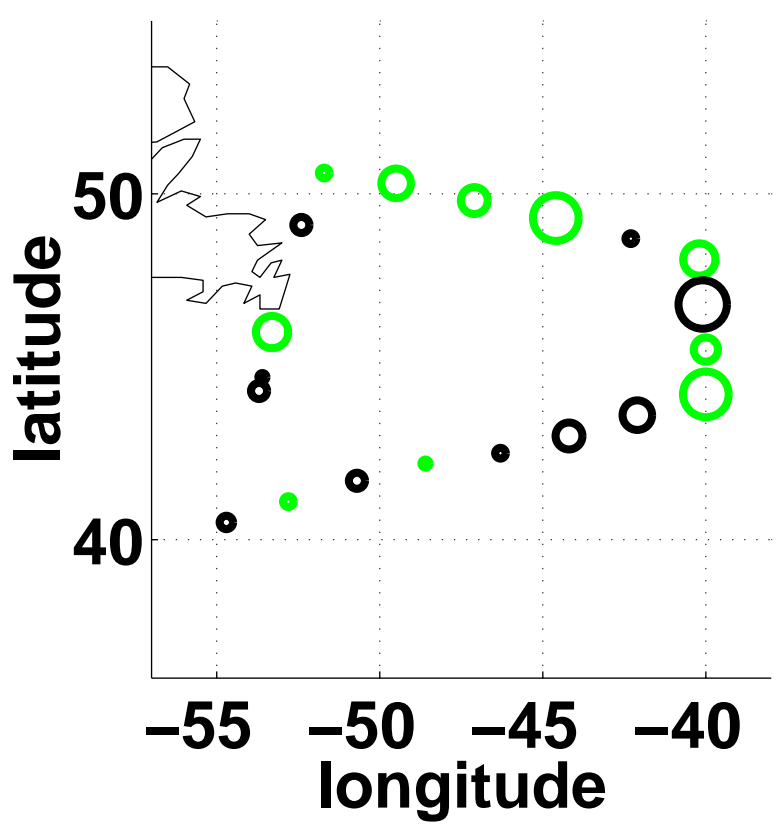

(d)

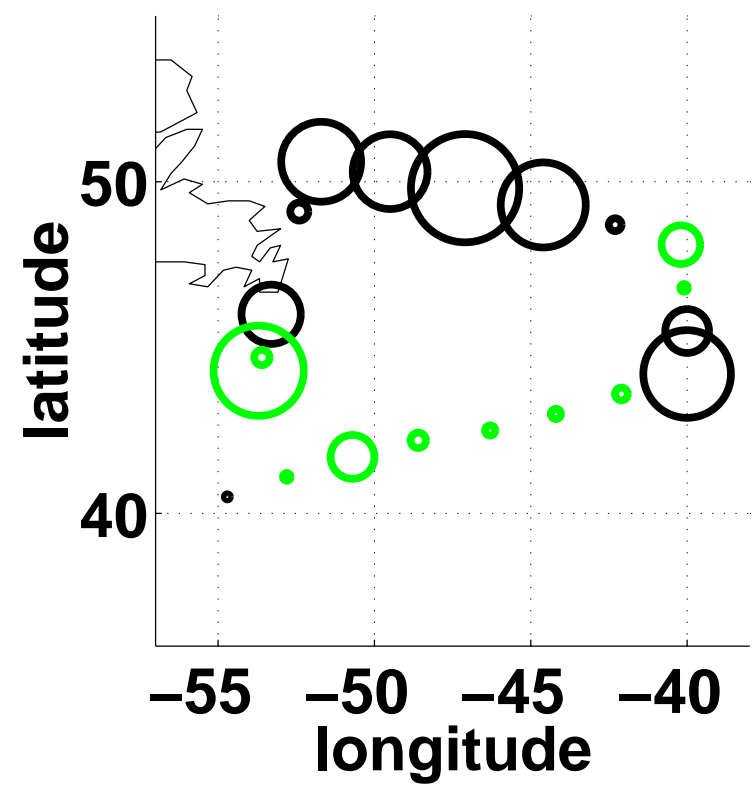

Fig. 8. GIV flight for IOP17. Modification of $S$ by targeted observations. Extremum of these modifications for temperature (a) and wind (b). Vertical integration of these modifications for temperature (c) and wind (d). A given size of the symbols corresponds to similar modifications in Figs. (a)-(b), and in Figs. (c)-(d). The black circles correspond to an increase in $S$, and the gray circles to a decrease in $S$.

be the most efficient sampling of a sensitive area, given the assimilation scheme used, and given the conventional observation network? There are two major problems for answering such a question. The first problem is related to the unknown size of the innovation vector (difference between the background field and the observations). This work has shown that observations located in a strong sensitivity area have a strong impact. However, observations can have a significant impact, even if they are not located at an extremum of the sensitivity, but embedded in significant values of the innovation. This clearly implies that not only the sensitivity field should be taken into account, but also the estimated innovation at the observation location. This point will be studied in a future work. The second problem is related to the definition of an optimal sampling of the sensitive area. The optimization of a flight plan will be a complicated problem: one wants to find the matrix $\mathbf{H}_{t}$ that maximizes $\nabla_{\mathbf{y} t} S$. A suboptimal solution is to find the optimal location of targeted observations in a sequential manner. In this case, one identifies the best location for one targeted 


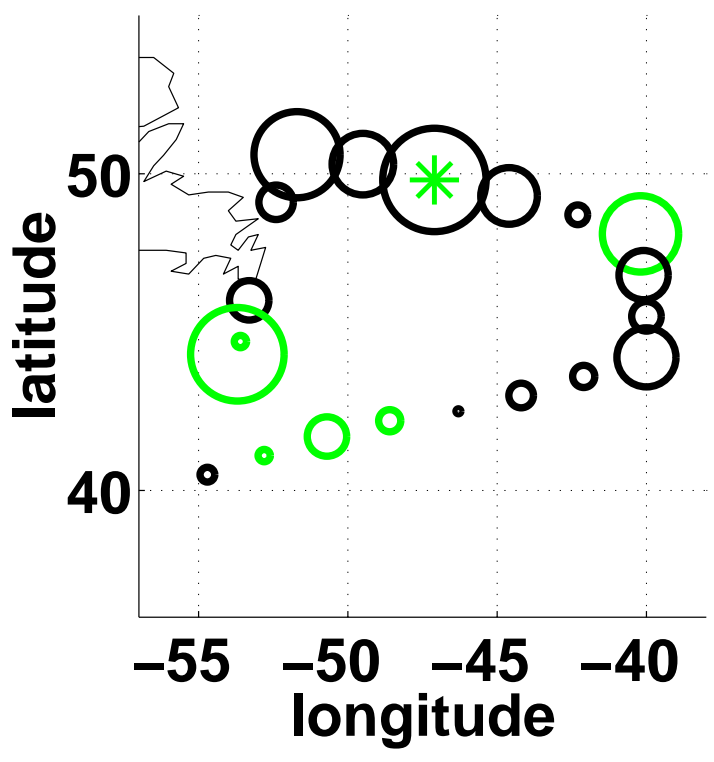

Fig. 9. GIV flight for IOP17. Same as in Fig. 8 for the total impact per dropsonde.

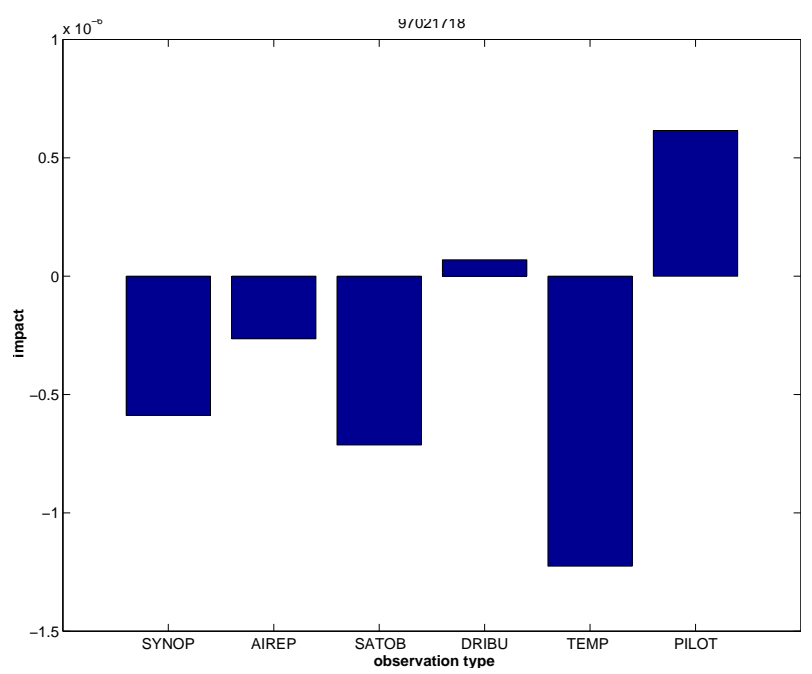

Fig. 10. GIV flight for IOP17. Modification of the forecast aspect $S$ for different kinds of observations: SYNOP (surface measurements), AIREP (commercial aircrafts data), SATOB (satellite data), DRIBU (buoy data), TEMP (targeted dropsondes) and PILOT (altitude wind data). The studied data are in a window of $110 \mathrm{~W} / 0$ and $20 \mathrm{~N} / 70 \mathrm{~N}$.

observation at a time (the problem becomes in this way a scalar optimization problem). Once this location has been found, one identifies the best location for the next targeted observation, given that all previous targeted observations have been taken into account. This method is very efficient, but is only a suboptimal method. This new tool should be tested under real conditions, for example, during the preliminary phase of the future THORPEX field experiment (The Hemispheric Observing system Research and Predictability EXperiment; http://box.mmm.ucar.edu/uswrp/fieldprojects/fieldprojects.html).

\section{Appendix A Effect of the covariances}

\section{A1 Formalism}

The goal of this appendix is to show the relationship between the vertical extension of the sensitivity to observations and the vertical extent of the error covariances of the background field. We chose to illustrate this point with a few low dimensional examples. But first, it is necessary to clarify a few notations (see also Baker and Daley, 2000).

\section{A1.1 Mathematical notations}

Prior to any result, we remain the readers of the principle of $\mathbf{K}^{T}$ that is used in the computation of the sensitivity to the observations, and which performs a change from model to observation space.

\begin{tabular}{ccc}
$\begin{array}{c}\text { Observation } \\
\text { Space }\end{array}$ & & $\begin{array}{c}\text { Initial Conditi } \\
\text { Model Sp }\end{array}$ \\
\hline $\boldsymbol{y}$ & & $\boldsymbol{x}_{a}$ \\
\cline { 2 - 3 }$\nabla_{\mathbf{y}} S$ & $\mathbf{A H}^{T} \mathbf{R}^{-1}$ & \\
& $\mathbf{K}^{T}$ & $\nabla_{\mathbf{x}} S$
\end{tabular}

Moreover, it is worth recalling that $\mathbf{A}$ can be calculated following two equivalent formulae:

$$
\begin{aligned}
\mathbf{A} & =\left(\mathbf{B}^{-1}-\mathbf{H}^{T} \mathbf{R}^{-1} \mathbf{H}\right)^{-1} \\
& =\mathbf{B}-\mathbf{B H}^{T}\left(\mathbf{R}+\mathbf{H B} \mathbf{H}^{T}\right)^{-1} \mathbf{H B} .
\end{aligned}
$$

Let us consider the state vector $\boldsymbol{x}$ of the model we choose to work with. Let $m$ be its dimension. $\boldsymbol{x}$ can be written as:

$\boldsymbol{x}=\left[\begin{array}{lllll}x_{1} & x_{2} \ldots & x_{i} & \ldots & x_{m}\end{array}\right]^{T}$.

From a similar point of view, let us consider the observation vector $\boldsymbol{y}$ with dimension $n$. Practically, with the NWP model, $n$ is smaller than $m$ :

$\boldsymbol{y}=\left[\begin{array}{llllll}y_{1} & y_{2} & \ldots & y_{k} & \ldots & y_{n}\end{array}\right]^{T}$.

$\mathbf{A}$ and $\mathbf{B}$ are both $m \times m$ symmetric matrices. $\mathbf{R}$ is a $n \times n$ matrix which we assure to be diagonal. This hypothesis is quite a strong one: the measurement errors are supposed to be uncorrelated between each other, even if the same dropsonde is used to perform them. This hypothesis is no longer valid for satellite observations because errors in observations performed by a given satellite are well-known to be correlated. Moreover, this assumption of independence between observation errors is particularly true between distinct observation types or platforms. However, we allow the variances of observation errors to vary according to the location and the type of observation. This agrees with the reality: the same dropsonde is considered to provide less accurate measurements (higher variances of the errors) at the top and bottom of the atmosphere than at mid-levels. In other words, 
and as it is done in the operational assimilation scheme, the observations errors are uncorrelated and $\mathbf{R}$ is kept diagonal.

Let $a_{i j}$ and $b_{i j}$ be the components corresponding to the $i^{\text {th }}$ column and the $j^{\text {th }}$ line of $\mathbf{A}$ and $\mathbf{B}$, respectively. $\sigma_{k}^{o}$ is the $k^{t h}$ element of the diagonal of $\mathbf{R}$.

\section{A1.2 The simplified context}

To give a more meteorological meaning to the cases studied here, we will perform some numerical calculations within a simplified context. The different components or parameters, $x_{j}$ of the model state vector $\boldsymbol{x}$, correspond to the different levels ( $m$ levels) of the atmosphere. Practically, we take $m$ to be 50. In our experiments, we test different formulations of the operators that are used in Eq. (A1.1) to show the effect of the structure functions depicted by $\mathbf{B}$ in the sensitivity computations from different points of view. Two different kinds of variance and covariance descriptions for the errors of background and observations are used. The first one depicts a simplified situation in which the covariances of the background errors are parameterized by a decreasing exponential function of the distance between the model levels. The range of the decrease of the covariances as well as the variance of the background errors remains constant, and equal to one at all levels. The variances of the observation errors are equal to 1 . This configuration is named "flat" hereafter. In the second one, called a "curved" configuration, we consider a slightly more complicated matrix B. The covariances follow an exponential function as before, but the variances of the background errors vary according to the level, following a quadratic formulation (the variance is greater near the ground and aloft than at mid-levels).

\section{A2 Identical model and observation space}

This case does not need to be illustrated with numerical results. We will only use a mathematical formalism. Let us consider that each model grid point is an observation point. In this particular case, let us consider that the interpolation scheme is the identity $(\mathbf{H}=I)$. Using the fact that $\mathbf{A}$ and $\mathbf{R}$ are self-adjoint, we have the following property:

$\mathbf{K}=\mathbf{A} \cdot \mathbf{H}^{T} \cdot \mathbf{R}^{-1}=\mathbf{A} \cdot \mathbf{R}^{-1}$

$\mathbf{K}^{T}=\mathbf{R}^{-T} \cdot \mathbf{A}=\mathbf{A} \cdot \mathbf{R}^{-1}=\mathbf{K}$

It can be noted that the fact that $\mathbf{R}$ is a diagonal matrix implies that it can permuted with $\mathbf{A}$.

$\mathbf{K}$ and $\mathbf{K}^{T}$ correspond to the same operator; the effect of the structure functions contained in them is then identical, and the effect of $\mathbf{K}$ on the innovation (in the forward analysis process) is the same as the effect of $\mathbf{K}^{T}$ on the computation of the sensitivity to observations.

\section{A3 Single observation case without interpolation}

Now let us consider a single observation measurement which is performed at the location of a model grid-point $i$. First, we assume that the interpolation scheme is very simple:

$$
\mathbf{H}=[0 \ldots 010 \ldots 0] .
$$

This means that the information contained in the observation is only transfered to the grid-point $i$. Using the second formulation of $\mathbf{A}$ (Eq. A1), this configuration of observations leads to the following expression for the component of $\mathbf{A}$ :

$\left[a_{j k}\right]=\left[b_{j k}-\frac{b_{j i} \cdot b_{i k}}{\sigma_{i}^{o}+b_{i i}}\right]$.

\section{A3.1 Forward case: analysis of this single observation}

Let $\boldsymbol{d}$ be the innovation vector, i.e. $\boldsymbol{d}=\left(\boldsymbol{y}-\mathbf{H} \boldsymbol{x}_{b}\right)$. The analyzed field is given by $\boldsymbol{x}_{a}=\boldsymbol{x}_{b}+\mathbf{A} \mathbf{H}^{T} \mathbf{R}^{-1} \boldsymbol{d}$. Given the formulation of $\mathbf{H}, \boldsymbol{d}$ simplifies to its single component: $\boldsymbol{d}=$ $d_{i}=y_{i}-\left[\boldsymbol{x}_{b}\right]_{i}$, and the assimilation of this single observation leads to the following analysis increment $\left(\delta \boldsymbol{x}_{a}=\boldsymbol{x}_{a}-\boldsymbol{x}_{b}\right)$ :

$$
\begin{aligned}
\delta \boldsymbol{x}_{a}= & {\left[\left(b_{i k}-\frac{b_{i i} \cdot b_{i k}}{\sigma_{i}^{o}+b_{i i}}\right) \frac{\boldsymbol{d}}{\sigma_{i}^{o}}\right]_{k=1, m} } \\
= & {\left[\frac{\boldsymbol{d}}{\sigma_{i}^{o}+b_{i i}} b_{i k}\right]_{k=1, m} . }
\end{aligned}
$$

Considering that $\boldsymbol{d} / \sigma_{i}^{o}+b_{i i}$ is a normalization factor $(i$ is defined as the observation position in $\mathbf{H}), \delta \boldsymbol{x}_{a}$ appears to be the $i^{\text {th }}$ column of $\mathbf{B}$ (i.e. $\left.\left[b_{i k}\right]_{k=1, m}\right)$, weighted by $d /\left(\sigma_{i}^{o}+\right.$ $\left.b_{i i}\right)$.

The single observation is simulated at the thirtieth level with a measurement that makes the innovation be equal to half of the standard deviation of the observation error: $\boldsymbol{d}=$ $\sigma^{o} / 2$. Figure A 1 shows the analysis increment (bold line) for both a "flat" formulation (a) and a "curved" formulation (b). For the values of the statistics used in this experiment, we find the maximum increment to be $1 / 4$, as it can be estimated from the Eq. (A8) with $k=30, \sigma^{o}=b_{k k}=1$ and $\boldsymbol{d}=$ $1 / 2$. As explained in the main part of the paper in this single observation context, the analysis increment (bold curve in Fig. A1 represents the structure function associated with the assimilation scheme used.

\section{A3.2 Sensitivity to a single observation}

Now let us focus on the adjoint problem of the assimilation of a single observation. First of all, we recall hereafter the formula that gives the sensitivity of a forecast aspect $S$ to the observations:

$\nabla_{\mathbf{y}} S=\mathbf{R}^{-1} \mathbf{H A} \nabla_{\mathbf{x}} S$

where $\nabla_{\mathbf{x}} S$ is the sensitivity of $S$ to the initial conditions of the forecast (namely $\boldsymbol{x}_{a}$ ). Let $\left[g_{k}\right]$ be the $k^{t h}$ component of $\nabla_{\mathbf{x}} S=\left[g_{k}\right]_{k=1, m}$. For a single observation experiment, the 
(a)

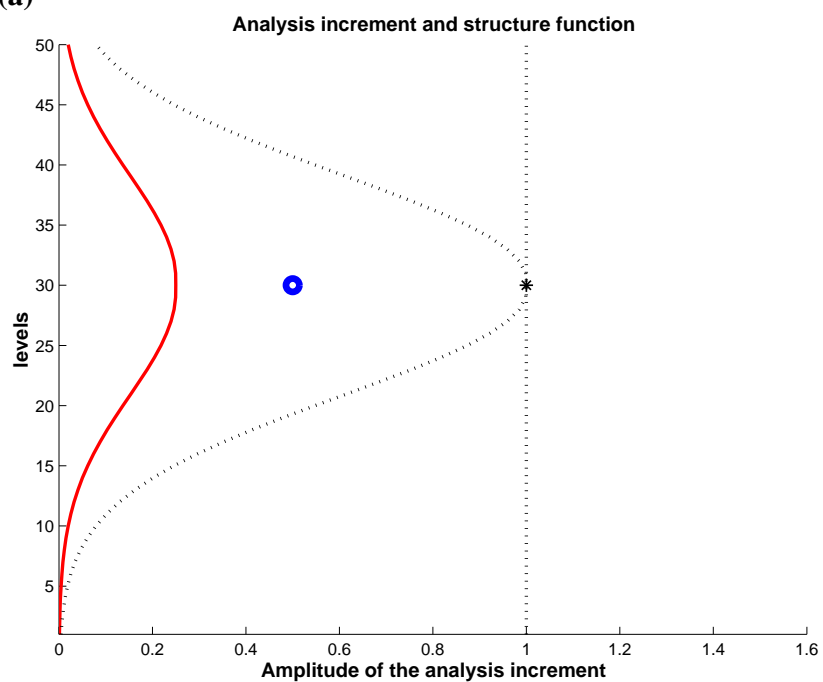

(b)

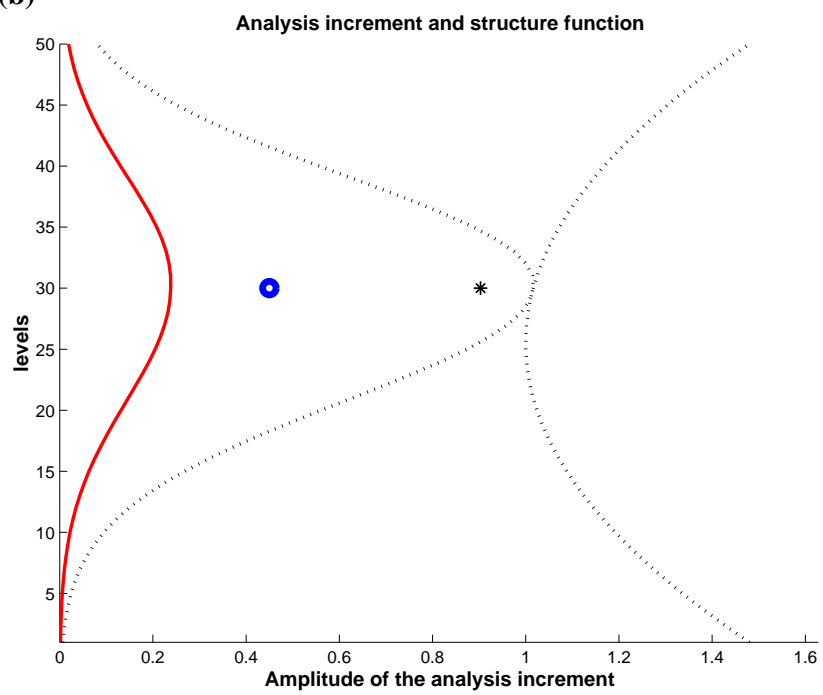

Fig. A1. Increment of assimilation for the "flat" formulation (a) and the "curved" formulation (b). The circle shows the value of the innovation vector $\boldsymbol{d}$ and location of the single observation in the column of atmosphere. The bold curve shows the analysis increment corresponding to the assimilation of this single observation. The dotted line on the left shows the covariances of the background observed parameter with all the other model parameters $b_{i j}$. The dotted line on the right shows the variances $b_{i i}$ on the vertical. Finally, the star shows the value of $\sigma^{o}$.

sensitivity to the observation is a scalar. If $\nabla_{y_{i}} S$ depicts this scalar,

$\nabla_{y_{i}} S=\sum_{k=1}^{m} \frac{a_{k i} \cdot g_{k}}{\sigma_{i}^{o}}$

which can be rewritten using the Eq. (A7) to replace $a_{k i}$, and the fact that $\mathbf{B}$ is symmetric (i.e. $b_{j k}=b_{k j}$ ):

$\nabla_{y_{i}} S=\sum_{k=1}^{m} \frac{b_{k i} \cdot g_{k}}{\sigma_{i}^{o}+b_{i i}}$
Eventually, we can isolate the scaling factor $1 /\left(\sigma_{i}^{o}+b_{i i}\right)$ to write:

$\nabla_{y_{i}} S=\frac{1}{\sigma_{i}^{o}+b_{i i}} \sum_{k=1}^{m} b_{k i} . g_{k}$.

This last Eq. (A12) shows that the sensitivity to a single observation is not only influenced by what happens at point $i$, but also by all the neighbouring grid-points, according to the structure function $\left[b_{i k}\right]_{k=1, m}$. Now, let us imagine a sensitivity to initial conditions, $\nabla_{\mathbf{x}} S$, with a significant extremum with respect to a single grid-point. This means that the studied forecast aspect is highly sensitive to what is present in the analysis state vector, $\boldsymbol{x}_{a}$, at a single level $k$, the sensitivity to the other level being quite negligible. The sensitivity to initial conditions can then be written:

$\nabla_{\mathbf{x}} S=\left[\begin{array}{lllllll}0 & \ldots & 0 & g_{k} & 0 & \ldots & 0\end{array}\right]^{T}$.

In this case, the sensitivity of $S$ to the single observation is given by:

$\nabla_{y_{i}} S=\frac{b_{k i} \cdot g_{k}}{\sigma_{i}^{o}+b_{i i}}$.

If we test different locations for this single observation, i.e. if we perform $m$ separate experiments corresponding to the observation located on one of the $m$ levels of the model (the level of measurement $i$ will vary from 1 to $m$ ), we can visualize the structure function $\left[b_{i k}\right]_{i=1, m}$. This is especially true if the scaling factor $1 /\left(\sigma_{i}^{o}+b_{i i}\right)$ is uniform, i.e. if the variance of both observation and background errors are uniform.

To illustrate the statements given above, we performed 50 experiments, thus testing 50 different locations for the measurement. We chose to place a unit peak of sensitivity to the initial conditions at the thirtieth level, i.e. is setting $g_{30}=1$ and $g_{i}=0$ if $i \neq 30$ in Eq. (A13).

Figure A2a depicts the "flat" situation where the variance of background statistics are constant in the vertical. From this choice arises the fact that the curve depicted by the successive 50 experiments corresponds to half of the structure function $\left[b_{i k}\right]_{i=1, m}(k=30$ here). This effect becomes obvious when comparing the stepped curve to the left-hand dotted one in Fig. A2a, which describes the successive sensitivities to the varying single observation. In Fig. A2b, the variances are in the "curved" configuration. In this more general context, we again verify the theoretical result which emphasizes the spreading effect of the structure functions contained in $\mathbf{B}$.

\section{A4 Single observation case with interpolation}

In this subsection, we chose to compare the spreading effects due to $\mathbf{B}$ on the one hand, and due to the interpolation scheme contained in $\mathbf{H}$ on the other hand. In this case, the more complicated operator $\mathbf{H}$ leads to a heavier mathematical formalism of little interest. It seems more useful to directly show numerical results. 
(a)

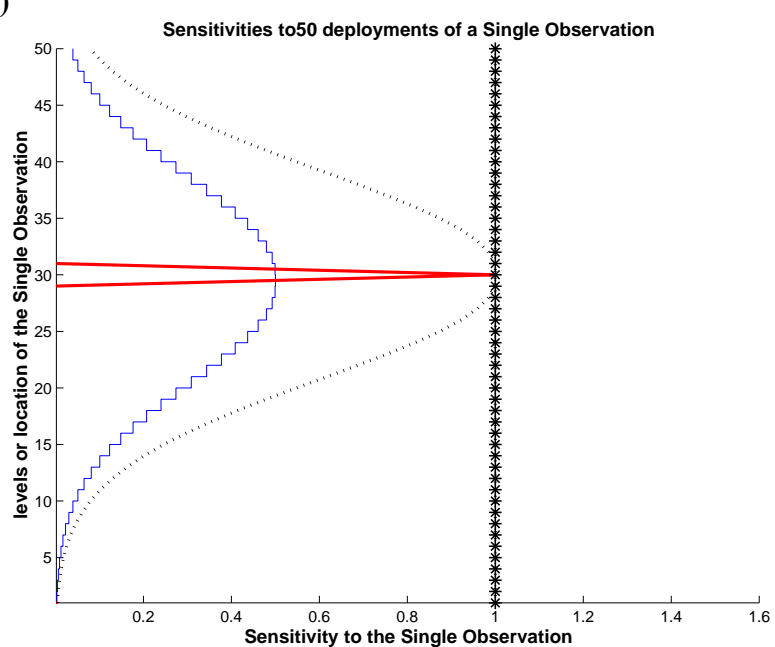

(b)

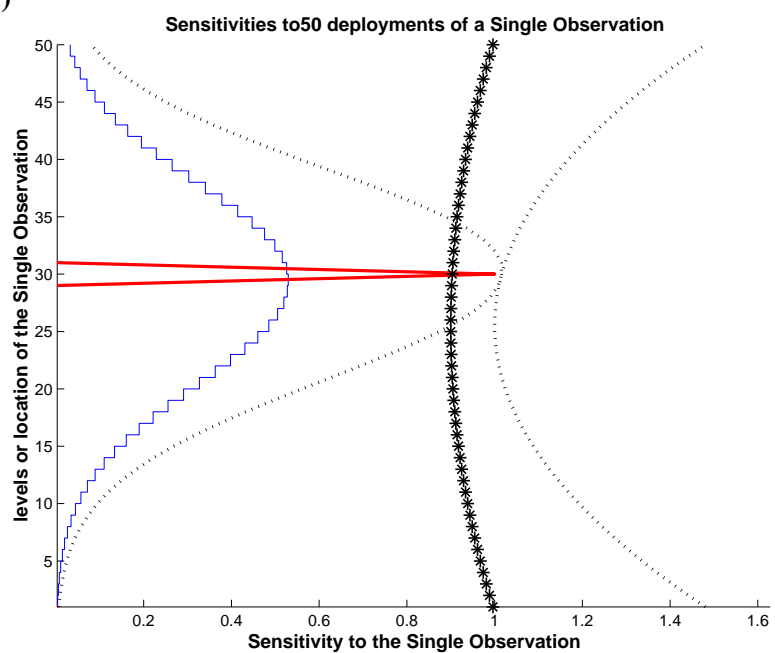

Fig. A2. Sensitivity to a single observation for the "flat" formulation (a) and the "curved" formulation (b). The stepped line shows the value of the sensitivity for each location of the observation at the corresponding level. The bold solid curve shows the peak of sensitivity to the initial conditions which is the same for all the experiments. The dotted line on the left shows the covariances of the background observed parameter with all the other model parameters $b_{i j}$. The dotted line on the right shows the variances $b_{i i}$ on the vertical. Finally, the stars show the different values of $\sigma^{o}$ along the 50 experiments.

We implemented a linear interpolation scheme that uses $p=2$ surrounding levels. Five contiguous levels are involved in the interpolation: 2 levels above, 2 below and the level of observation. These results are plotted in Fig. A3. Comparing Figs. A2b and A3, it appears that the interpolation scheme has quite a negligible influence. The reason for this is that the range of the interpolation scheme is by far much less than the range inherent in the background covariances.

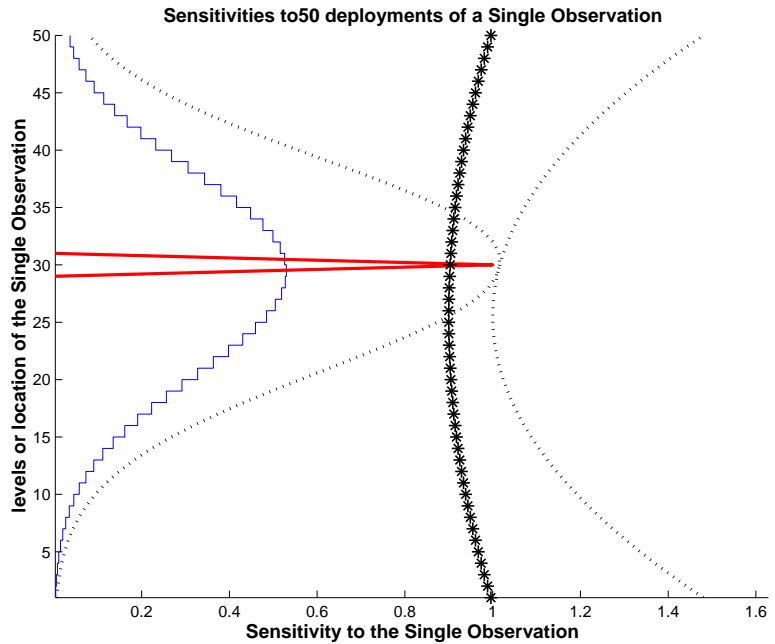

Fig. A3. General case: the curves represent the same quantities as in the Fig. A2.

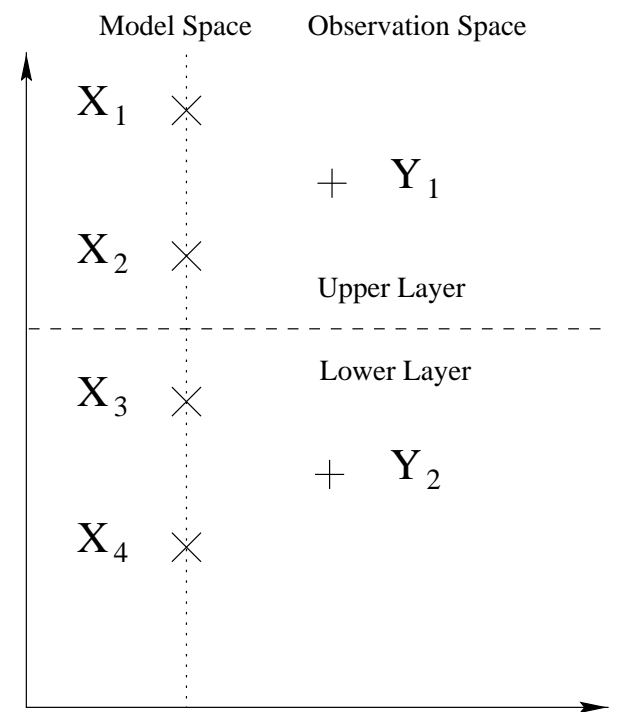

Fig. A4. Model space gridpoints and corresponding observations for the first simple experiment configuration.

\section{A5 Several types of observation}

In this subsection, we shall give consideration to the ability of the covariances of the background errors to spread sensitivity to initial conditions from one atmospheric region to the neighbouring ones. For this purpose, we will consider that the model domain is divided into $k$ regions. Taking into account the non-spreading property of the interpolation scheme, $\mathbf{H}$ can be written as:

$\mathbf{H}=\left[\begin{array}{cccc}\mathbf{H}_{1} & 0 & \ldots & 0 \\ 0 & \mathbf{H}_{2} & \ldots & 0 \\ \vdots & \vdots & \ddots & \vdots \\ 0 & 0 & \ldots & \mathbf{H}_{k}\end{array}\right]$ 
To simplify the writing, we consider in the following that there are two regions in the atmosphere $(k=2)$, and that each one is observed with a network of $n_{k}$ observations (we have $n=n_{1}+n_{2}$ ), see Fig. A4. The interpolation scheme in $\mathbf{H}$ is assumed not to be able to transfer any information from one region to another. With this strong assumption, we want to show that the covariances, or $\mathbf{K}$, are able to do so on their own.

According to the previous hypothesis, let us calculate $\mathbf{K}$. The covariance errors matrices can be written as

$\mathbf{R}=\left[\begin{array}{cc}\mathbf{R}_{1} & 0 \\ 0 & \mathbf{R}_{2}\end{array}\right]$ and $\mathbf{B}=\left[\begin{array}{ll}\mathbf{B}_{11} & \mathbf{B}_{21} \\ \mathbf{B}_{12} & \mathbf{B}_{22}\end{array}\right]$.

Then, using Eq. (A1), $\mathbf{A}$ can be rewritten as

$\mathbf{A}=\mathbf{B B H}^{T}$

$$
\cdot\left[\begin{array}{cc}
\left(\mathbf{R}_{1}+\mathbf{H}_{1} \mathbf{B}_{11} \mathbf{H}_{1}^{T}\right)^{-1} & \left(\mathbf{H}_{1} \mathbf{B}_{21} \mathbf{H}_{2}^{T}\right)^{-1} \\
\left(\mathbf{H}_{2} \mathbf{B}_{12} \mathbf{H}_{1}^{T}\right)^{-1} & \left(\mathbf{R}_{2}+\mathbf{H}_{2} \mathbf{B}_{22} \mathbf{H}_{2}^{T}\right)^{-1}
\end{array}\right]
$$

.HB.

At that point two hypothesis can be checked. They are based on two cases where the background errors present in the both regions are either correlated or not. The hypothesis of nocorrelation implies that both block $\mathbf{B}_{21}$ and $\mathbf{B}_{12}$ are equal to zero, and in that case, the Eq. (A17) yields:

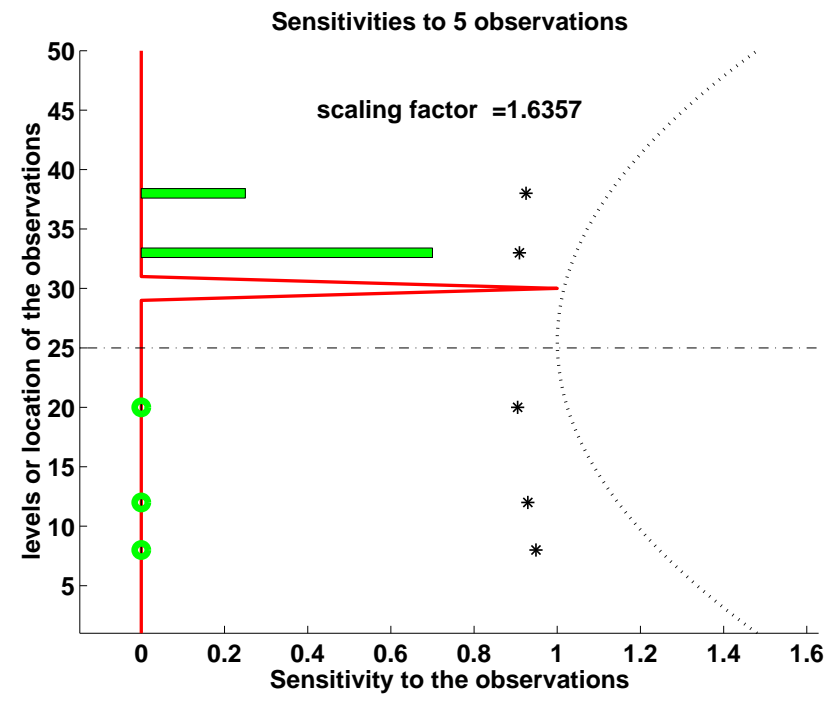

Fig. A5. Sensitivity to 5 observations spread in the two layers (separated by the chain line) of the atmosphere column. The horizontal bars show the scaled sensitivity to the observation located at the corresponding level (bold circles correspond to null sensitivity). Bold line, dashed lines and the stars (all non-scaled) have the same meaning as in the Fig. A1.
Eventually, the adjoint of the gain operator is also block diagonal:

$\mathbf{K}^{T}=\left[\begin{array}{cc}\mathbf{K}_{1}^{T} & 0 \\ 0 & \mathbf{K}_{2}^{T}\end{array}\right]$.

If one considers a forecast aspect which is sensitive to the initial conditions in a single region, only observations located in that region will lead to significant sensitivities to observations. To illustrate this statement, let $\nabla_{\mathbf{x}} S$ be as follows:

$\nabla_{\mathbf{x}} S=\left[\begin{array}{ll}g_{1} & 0\end{array}\right]^{T}$.

Then, the sensitivity to observations writes:

$\nabla_{\mathbf{y}} S=\left[\begin{array}{ll}\mathbf{K}_{1}^{T} g_{1} & 0\end{array}\right]^{T}$.

It appears that the forecast aspect is rather sensitive to observations belonging to the "lower" region. This result is illustrated by Fig. A5 which was produced with the same numerical package as before, with $k=2$ regions, $n=5$ observations $\left(n_{1}=2\right.$ and $\left.n_{2}=3\right)$. The level of shift between the two regions is $h=25$. We use the "curved" configuration of $\mathbf{B}$ that has been modified to keep zero covariances in both extra diagonal blocks, i.e. that some correlations still exist inside a given region, whereas there are no correlations between the two regions. One can note that there is no transfer of information from the region where the gradient to initial conditions $\nabla_{\mathbf{x}} S$ is significant to the other region (bold circle with null sensitivity). In the case where the background errors present in both regions are correlated, the theoretical formulation of (A17) becomes heavy to handle : $\mathbf{B}_{21}$ and $\mathbf{B}_{12}$ are not zero. Therefore, $\mathbf{A}$ is a full $m \times m$ matrix, and $\mathbf{K}^{T}$ is not block diagonal:

$\mathbf{K}^{T}=\left[\begin{array}{ll}\mathbf{K}_{1}^{T} & \mathbf{K}_{21}^{T} \\ \mathbf{K}_{12}^{T} & \mathbf{K}_{2}^{T}\end{array}\right]$.

Using the same gradient as stated previously (see Eq. A20), the sensitivity to observations then writes:

$\nabla_{\mathbf{y}} S=\left[\begin{array}{ll}\mathbf{K}_{1}^{T} g_{1} & \mathbf{K}_{12}^{T} g_{1}\end{array}\right]^{T}$.

As written in the previous paragraph, let us illustrate this case with a numerical example given in Fig. A6. For this case we again use the "curved" configuration of $\mathbf{B}$. One can note that the sensitivity present in the upper region can easily be spread to the lower region due to the correlation present in $\mathbf{B}$. 


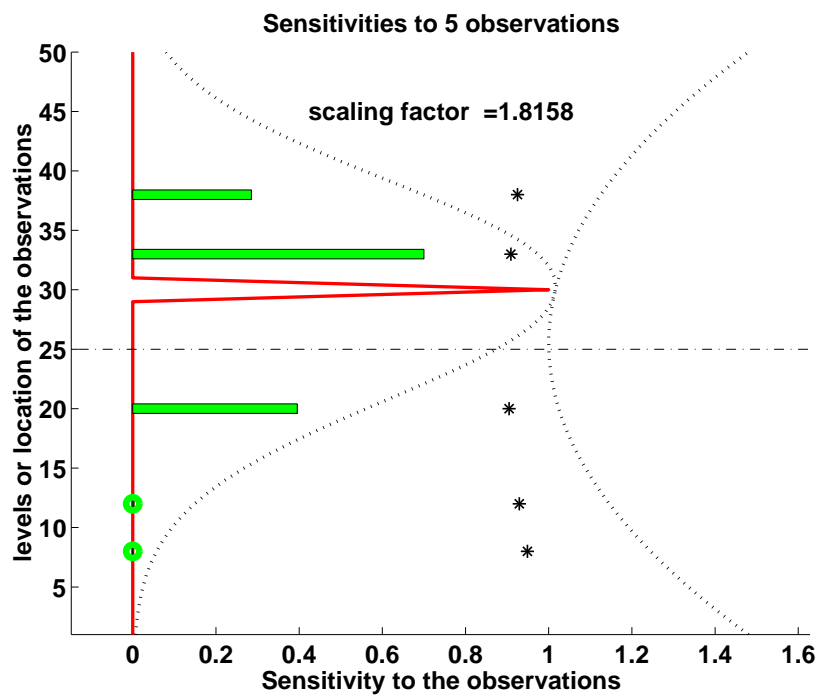

Fig. A6. Same as Fig. A1 with some correlations between all the levels.

Acknowledgements. The authors address special thanks to F. Bouttier for very helpful discussions about the role of the assimilation scheme and about the use of the sensitivity to observations. The practical implementation of this method within the ARPEGE model was possible thanks to the work of Mike Fisher from ECMWF; we thank him for his useful advice. We are particularly grateful to Philippe Lopez for helpful comments on the first version of this article. Our colleagues from the GMME/RECYF team are also strongly acknowledged for their daily scientific and technical support.

Eventually, we are keen on thanking Craig Bishop, Jim Hansen and reviewer B for their careful review of the preliminary version of this paper and their fruitful remarks.

\section{References}

Baker, N. L. and Daley, R., Observation and background adjoint sensitivity in the adaptive observation targeting problem, Quart. J. Roy. Meteor. Soc. , 126 , 1431-1454, 2000.

Bergot, T., Adaptive Observations during FASTEX : A systematic survey of upstream flights, Quart. J. Roy. Meteor. Soc., 125, 3271-3298, 1999.

Bergot, T., Influence of assimilation scheme on the efficiency of adaptive observations. Quart. J. Roy. Meteor. Soc., 127, 635661, 2001

Bergot, T., Hello, G., Joly, A., and Malardel, S., Adaptive Observations : a feasibility study. Mon. Weather Rev., 127, 743-765, 1999.

Bishop C. H., Etherton, B. J., and Majumdar, S. J., Adaptive sampling with the ensemble transform Kalman filter. Part I : Theo- retical aspects, Mon. Weather Rev., 129, 420-436, 2001.

Cammas, J.-P., Pouponneau, B., Desroziers, G., Santurette, P., Joly, A., Arbogast, P., Mallet, I., Caniaux, G., and Mascart, P., FASTEX IOP17 cyclone: introductory synoptic study with field data, Quart. J. Roy. Meteor. Soc., 125, 3393-3414, 1999.

Courtier, Ph., Freydier, C., Geleyn, J. F., Rabier, F., and Rochas, M., The ARPEGE project at Meteo-France, ECMWF, workshop on numerical methods in atmospheric models, Reading, UK, 9-13 Sept, 193-231, 1991.

Doerenbecher, A. and Bergot, T., (currently in progress) : Sensitivity to observations applied on FASTEX targeted observations. Quart. J. Roy. Meteor. Soc., in preparation, 2001.

Emanuel, K., Raymond, D., Betts, A., Bosart, L., Bretherton, C., Droegemier, K., Farrell, B., Fritsch, J.-M., Houze, R., Lemone, M., Lilly, D., Rotunno, R., Shapiro, M., Smith, R., and Thorpe, A., Report of the first prospectus development team of the US weather research-program to NOAA and the NSF, Bull. Amer. Meteor. Soc., 76-7, 1194-1208, 1995.

Errico, R., What is an adjoint model?, Bull. Amer. Meteor. Soc., 78, 2577-2591, 1997.

Fisher, M. and Courtier, P., Estimating the covariance matrices of analysis and forecast error in variational data assimilation, ECMWF Tech. Memo., 220, 1995.

Ghil, M. and Malanotte-Rizzoli, P., Data assimilation in meteorology and oceanography, Adv. Geophys., 33, 141-266, 1991.

Ide, K., Courtier, P., Ghil, M., and Lorenc, A. C., Unified Notation for Data Assimilation : Operational, Sequential and Variational, J. Meteorol. Soc. Japan, 75 1B, 181-189, 1997.

Joly, A. and co-authors, Overview of the field phase of the Fronts and Atlantic Storm Track Experiment (FASTEX) project, Quart. J. Roy. Meteor. Soc., 125, 3131-3164, 1999.

Langland, R. H. and co-authors, The North Pacific Experiment 8 (Norpex-98): targeted observations for improved North American Weather Forecasts, Bull. Amer. Meteor. Soc., 80, 13631384, 1999.

Lorenc, A. C., Analysis method for numerical weather prediction, Quart. J. Roy. Meteor. Soc., 112, 1177-1194, 1986.

Morss, R. E., Adaptive observations: idealized sampling strategies for improving numerical weather prediction, $\mathrm{Ph}$. D. Thesis, Massachusetts Institute of Technology, Cambridge, MA, 225pp, 1999.

Rabier, F., Klinker, E., Courtier, P., and Hollingsworth, A., Sensitivity of forecast errors to initial conditions, Quart. J. Roy. Meteor. Soc., 122, 121-150, 1996.

Snyder, C., Summary of an informal workshop on adaptive observations and FASTEX, Bull. Amer. Meteor. Soc., 77, 953-961, 1996.

Szunyogh, I., Toth, Z., Morss, R., Majumdar, S., Etherton, B. J., and Bishop, C. H., The effect of targeted dropsonde observations during the 1999 Winter Storm Reconnaissance Program, Mon. Weather Rev., 128, 3520-3537, 2000.

Thépaut, J.-N., Courtier, P., Belaud, G., and Lemaitre, G., Dynamical structure functions in a four-dimensional variational assimilation: a case study. Quart. J. Roy. Meteor. Soc., 122, 535, 561, 1996. 